\title{
Monoamines as Drug Targets in Chronic Pain: Focusing on Neuropathic Pain
}

\author{
Lidia Bravo ${ }^{1,2,3}$, Meritxell Llorca-Torralba' ${ }^{1,2,3}$, Esther Berrocoso ${ }^{2,3,4}$ and \\ Juan Antonio Micó ${ }^{1,2,3 *}$
}

${ }^{1}$ Neuropsychopharmacology and Psychobiology Research Group, Department of Neuroscience, University of Cádiz, Cádiz, Spain, ${ }^{2}$ Instituto de Investigación e Innovación Biomédica de Cádiz, INiBICA, Hospital Universitario Puerta del Mar, Cádiz, Spain, ${ }^{3}$ Centro de Investigación Biomédica en Red de Salud Mental (CIBERSAM), Instituto de Salud Carlos III, Madrid, Spain, ${ }^{4}$ Neuropsychopharmacology and Psychobiology Research Group, Department of Psychology, University of Cádiz, Cádiz, Spain

\section{OPEN ACCESS}

Edited by:

Michael Costigan,

Boston Children's Hospital and

Harvard Medical School,

United States

Reviewed by:

Nick Andrews,

Harvard Medical School, United States

Ciaran J. Faherty,

Heron Therapeutics, Inc.,

United States

*Correspondence:

Juan Antonio Micó

juanantonio.mico@uca.es

Specialty section:

This article was submitted to

Neuropharmacology,

a section of the journal

Frontiers in Neuroscience

Received: 31 May 2019 Accepted: 08 November 2019 Published: 26 November 2019

Citation:

Bravo L, Llorca-Torralba M, Berrocoso E and Micó JA (2019) Monoamines as Drug Targets in Chronic Pain: Focusing on

Neuropathic Pain.

Front. Neurosci. 13:1268. doi: 10.3389/fnins.2019.01268
Monoamines are involved in regulating the endogenous pain system and indeed, peripheral and central monoaminergic dysfunction has been demonstrated in certain types of pain, particularly in neuropathic pain. Accordingly, drugs that modulate the monaminergic system and that were originally designed to treat depression are now considered to be first line treatments for certain types of neuropathic pain (e.g., serotonin and noradrenaline (and also dopamine) reuptake inhibitors). The analgesia induced by these drugs seems to be mediated by inhibiting the reuptake of these monoamines, thereby reinforcing the descending inhibitory pain pathways. Hence, it is of particular interest to study the monoaminergic mechanisms involved in the development and maintenance of chronic pain. Other analgesic drugs may also be used in combination with monoamines to facilitate descending pain inhibition (e.g., gabapentinoids and opioids) and such combinations are often also used to alleviate certain types of chronic pain. By contrast, while NSAIDs are thought to influence the monoaminergic system, they just produce consistent analgesia in inflammatory pain. Thus, in this review we will provide preclinical and clinical evidence of the role of monoamines in the modulation of chronic pain, reviewing how this system is implicated in the analgesic mechanism of action of antidepressants, gabapentinoids, atypical opioids, NSAIDs and histaminergic drugs.

Keywords: chronic pain, neuropathic pain, monoamines, antidepressants, noradrenaline, serotonin

\section{INTRODUCTION}

The monoaminergic system is implicated in the maintenance of homeostasis in the nervous system and among their distinct functions, monoamines regulate the endogenous pain system (Bannister et al., 2009). In terms of chronic pain, some recent contributions to the pharmacological arsenal of analgesics that target monoamines are selective inhibitors of serotonin and noradrenaline reuptake (SNRIs, e.g., duloxetine), and atypical opioids that combine their opioid activities with an effect on monoamines (e.g., tramadol and tapentadol). These drugs have opened the way to consider monoamines and their receptors as potential targets to develop innovative analgesics. Moreover, 
these drugs have also helped clarify the mechanism of action of some so-called atypical analgesics, the primary effects of which do not involve the monoaminergic system (i.e., tramadol, tapentadol, nefopam) even though their global efficacy as analgesics might well be driven by monoamines.

The most classic drugs that act directly on the monoaminergic system are antidepressants, drugs that were not originally designed as analgesics but that are currently considered first line analgesics for some types of neuropathic pain (Finnerup et al., 2005; Sindrup et al., 2005). Nevertheless, not all antidepressants have analgesic properties. For years, amitriptyline has been the most widely prescribed antidepressant but more recently, the SNRI duloxetine has been positioned as an analgesic antidepressant that can be used to treat peripheral diabetic neuropathic pain and fibromyalgia. By contrast, selective serotonin reuptake inhibitors (SSRIs) are not suitable analgesics to treat neuropathic pain. Atypical opioid drugs combine an opioid mechanism of action with another monoaminergic one, producing effective analgesia in several types of chronic pain, not only neuropathic pain (e.g., tramadol and tapentadol: Bravo et al., 2017; Coluzzi et al., 2017). In this review, we will present information from various preclinical and clinical studies that investigated the role of monoamines in chronic pain, as well as studies that assessed the mechanisms of action of actual and potential analgesics to treat different types of chronic pain.

\section{TYPES OF PAIN}

Pain is necessary for our survival as it is a process that helps protect us from danger and it serves as a short-term response to resolve injury. However, pain that persists for longer than 3 months is considered to be a pathological state known as chronic pain. Based on its etiology, pain is classified as: (i) nociceptive, caused by lesion or potential tissue damage that directly stimulates $\mathrm{C}$ and A $\delta$ fibers (e.g., somatic and visceral pain); (ii) inflammatory, caused by an inflammatory process (e.g., arthritis); (iii) neuropathic, caused by a lesion or disease affecting the somatosensory system (e.g., diabetic neuropathy, post-herpetic and neuralgic sciatic nerve injury). These three main types of pain can develop into chronic pain, although some particular types of chronic pain may not be properly covered by the categories of "nociceptive" or "neuropathic" pain, and they can be categorized as "nociplastic pain." This fourth type of pain is characterized by altered nociception in the absence of clear evidence of tissue damage or lesion, or of disease that affects the somatosensory system (International Association for Study of Pain, https://www.iasp-pain.org/PublicationsNews/ NewsDetail.aspx?ItemNumber=6862).

Regardless of its etiology, chronic pain is a public health problem that affects $20 \%$ of the adult population in Europe (Van Hecke et al., 2013). Chronic pain is one of the most frequent reasons for visits to a physician and its estimated financial cost exceeds $€ 200$ billion per annum in Europe and $\$ 150$ billion per annum in the United States (Tracey and Bushnell, 2009). One of the main problems of chronic pain is its inadequate management, most likely caused by ignorance of its biological roots. The monoaminergic system can modulate pain signaling and this system undergoes important alterations under conditions of chronic pain, contributing to its persistance. In fact, many types of chronic pain are now treated with medications that affect monoamines and as such, these will be discussed exhaustively in the present review.

\section{PAIN MODULATION BY MONOAMINES}

The monoaminergic system is an endogenous modulator of pain (Bannister and Dickenson, 2016). Peripheral nociceptive signals reach the spinal cord and the pain messages are relayed by ascending projections targeting the thalamus, the dorsal reticular nucleus (DRt), the rostral ventromedial medulla (RVM), and the midbrain periaqueductal gray (PAG) which integrates forebrain influences. The rostral projections from the thalamus target areas that several sub-regions in the cortex and the amygdala where both the sensory and affective components of pain are processed. In parallel, descending monoaminergic pain pathways, mainly from the Locus Coeruleus (LC) and RVM, modulate the ascending nociceptive information at the level of the spinal cord. These modulatory effects are mainly mediated by serotonin (5HT) and noradrenaline (NA), which may well represent potential targets for new analgesic drugs (see below).

\section{Noradrenergic Pain Modulation}

The noradrenergic system is mainly implicated in descending inhibitory pain with the noradrenergic descending projections to the spinal cord mainly arising from the A6 (LC), A5 and A7 (Howorth et al., 2009). The effects of NA are mediated by the $\alpha$ and $\beta$-NA adrenergic receptors, which are expressed widely in the central and peripheral nervous systems (CNS and PNS), with particularly strong expression in areas that directly participate in pain processing (e.g., PAG, RVM, thalamus, LC, prefrontal cortex and amygdala). There are various subtypes of adrenoceptors (ARs), a- and $\beta$-ARs ( $\alpha 1 \mathrm{~A}, \alpha 1 \mathrm{~B}, \alpha 1 \mathrm{D}, \alpha 2 \mathrm{~A}, \alpha 2 \mathrm{~B}$, and $\alpha 2 \mathrm{C}$; and $\beta 1, \beta 2$, and $\beta 3$ : Ruffolo and Hieble, 1994), with the $\alpha 2$-ARs most commonly associated with pain modulation. As we will describe here, these receptors may be suitable pharmacological targets to treat chronic pain.

\section{Peripheral Noradrenergic Modulation}

Noradrenaline is released locally by post-ganglionic sympathetic nerve fibers (Pertovaara, 2006). Many $\alpha$-ARs subtypes have been identified in the dorsal root ganglia (DRGs: $\alpha 1 \mathrm{~A}, \alpha 1 \mathrm{~B}, \alpha 1 \mathrm{D}$, $\alpha 2 \mathrm{~A}$, and $\alpha 2 \mathrm{C}$ ) and also on the nerve fibers distributed to the skin (Dawson et al., 2011) where peripheral nociception is locally modulated (Shi et al., 2000; Xie et al., 2001; Maruo et al., 2006). Although $\alpha 2$-AR agonists mainly produce analgesia via spinal and supraspinal action (Bernard et al., 1994; Buerkle and Yaksh, 1998; Asano et al., 2000), peripheral ARs also fulfill an important role in analgesia (Pertovaara, 2006). Thus, topical $\alpha 2$ agonist administration produces analgesia, an effect that is blocked by pretreatment with AR antagonists in acute and chronic pain rat models (Nakamura and Ferreira, 1988; Dogrul and Uzbay, 2004). Accordingly, topic administration of 
clonidine relieves hyperalgesia in patients with complex regional pain syndrome (Davis et al., 1991), indicating that peripheral stimulation of $\alpha 2$-ARs could relieve neuropathic pain. $\beta 2$-ARs are located on peripheral terminals and cell bodies of primary afferent nociceptors (Aley et al., 2001). Nevertheless, it remains unclear if activation of $\beta 2$-ARs is pro- or anti-nociceptive. Most preclinical studies have shown that peripheral $\beta 2$-AR stimulation by antidepressants induces anti-allodynic effects in neuropathic pain models (Bohren et al., 2013; Kremer et al., 2018), while intradermal injection of epinephrine produces hypersensitivity (Khasar et al., 1999; Parada et al., 2003).

\section{Central Noradrenergic Modulation}

Preclinical studies reported that chemical or electrical stimulation of the LC, the main noradrenergic nucleus, induces analgesia via the spinal cord that is blocked by spinal $\alpha 2$-AR antagonists (Pertovaara, 2006). The LC sends projections to all cortical regions, as well as to the basolateral amygdala (BLA), hippocampus, and ventral tegmental area (VTA), all of which express adrenergic receptors and are relevant structures in the context of pain (Loughlin et al., 1986; Sara and Bouret, 2012). Moreover, the LC express a large proportion of the $\alpha 2$-AR inhibitory auto-receptors that modulate NA release via these projections (Llorca-Torralba et al., 2016). Despite the prevalence of studies focusing on the LC in pain modulation, there is also evidence that other noradrenergic nuclei play a role in pain processing (Llorca-Torralba et al., 2016). Indeed, the A5 and A7 noradrenergic clusters provide significant NA input to the spinal cord, where the final balance between inhibitory and facilitatory modulation of pain takes place (Pertovaara, 2006; Bruinstroop et al., 2012). These findings provide evidence of the complex interaction between NA and pain. At present, the role of NA on spinal adrenergic receptors seems to be clearer than that its effects on supraspinal noradrenergic receptors.

\section{Serotonergic Pain Modulation}

Serotonin (5-HT, 5-hydroxytryptamine) has been widely related to pain modulation through peripheral and central actions. Unlike NA, which action seem to be more related to analgesia, 5HT acts on specific receptors that contribute to the maintenance of pain (Suzuki et al., 2004; Bannister and Dickenson, 2016).

\section{Peripheral Serotonergic Modulation}

Serotonergic nociceptive transmission at peripheral sensory nerves is mediated by several subtypes of 5-HT receptors (5HT1B, 5-HT1D, 5-HT2A, 5-HT2B, 5-HT3, 5-HT4, and 5-HT7) known to exist in DRGs (Pierce et al., 1996; Wu et al., 2001). Numerous studies have shown that peripheral administration of 5-HT increases the excitability of A $\delta$ and $C$ fibers, and DRG neurons (Michaelis and Janig, 1998; Lang et al., 2006), suggesting a pro-nociceptive role for 5-HT. Indeed, intradermal injection of 5-HT in rats produces dose-dependent hypersensitivity mediated by 5 -HT1A receptors (Taiwo et al., 1992). Moreover, peripheral injection of 5-HT2A, 5-HT3, and 5-HT7 antagonists produces an anti-nociceptive effect in a model of the formalin pain test (Rocha-González et al., 2005; Nakajima et al., 2009; GodinezChaparro et al., 2011). In clinical studies on healthy volunteers, intradermal injection of 5-HT produces burning pain (Lischetzki et al., 2001) and the hypersensitivity induced by 5-HT injections into the masseter muscle is antagonized by a 5-HT3 antagonist (Ernberg et al., 2000).

\section{Central Serotonergic Modulation}

Descending serotonergic pathways include the RVM, where the midline raphe magnus nucleus (RMN) can inhibit or facilitate descending pain (Fields, 2004). ON and OFF cells, respectively, in the RVM send descending inhibitory and excitatory fibers to the dorsal horn spinal cord neurons that control the spinal sensory transmission (Urban and Gebhart, 1999; Neubert et al., 2004). Thus, activation of the RVM by electrical stimulation or glutamate microinjection evokes the spinal release of 5-HT, inducing analgesia (Oliveras et al., 1975; Satoh et al., 1983; Aimone and Gebhart, 1986). The RVM receives projections from the periaqueductal gray (PAG) and the microinjection of opioids in RVM has an inhibitory effect on noxious stimulation, indirectly activating OFF cells and thereby reducing pain (Zorman et al., 1981; Jensen and Yaksh, 1989; Heinricher et al., 1994).

It was largely demonstrated that the effect of spinal 5HT is either inhibitory or facilitatory, depending on the 5HT receptor subtypes activated (Green et al., 2000; Millan, 2002; Dogrul and Uzbay, 2004; Suzuki et al., 2004). In pain modulation, 5-HT exerts excitatory effects via the 5-HT2 and 5HT3 receptors, yet an inhibitory effect is provoked by stimulation of 5-HT7 receptors (Obata et al., 2001; Viguier et al., 2013; Bannister et al., 2017). However, the 5-HT1A receptor could exert excitatory or inhibitory effects (Millan, 2002). Except to treat chronic migraine, no drugs targeting 5-HT receptors are used for chronic pain. Thus, central action of 5-HT clearly needs further investigation in order to understand how 5-HT modulates pain, and its implication as a possible analgesic agent in acute and chronic pain.

\section{Dopaminergic Pain Modulation}

The role of dopamine (DA) in pain modulation has received less attention than that of NA and 5-HT. The dopaminergic system is widely known to participate in mesocorticolimbic reward system, which arises from the VTA and modulates emotionrelated behavior (Baik, 2013). Interestingly, dopamine plays a role in the modulation of nociceptive transmission at both the spinal and supraspinal levels (Potvin et al., 2009). In the CNS, there are five subtypes of DA receptors (D1-D5), and the D1 and D2 receptors are those most strongly implicated in pain modulation, as witnessed in animal models (Millan, 2002). As such, activation of D2 receptors at the spinal level induces an anti-nociceptive effect (Magnusson and Fisher, 2000; Taylor et al., 2003; Ansah et al., 2007; Coffeen et al., 2008; Meyer et al., 2009), whereas stimulation of D1 receptors is pro-nociceptive (Gao et al., 2001; Yang et al., 2005; Kim et al., 2015).

Focal electrical stimulation of descending dopaminergic projections suppresses painful transmission at the spinal cord level, mainly through the A11 nucleus of the pericentral posterior hypothalamus and substantia nigra (Fleetwood-Walker et al., 1988). Thus, both acute and sustained noxious stimuli increase 
DA release to D2 receptors of spinothalamic and primary nociceptive neurons, eliciting an anti-nociceptive response (Millan, 2002). D1 receptor activation in the PAG or insular cortex has also been seen to attenuate pain-related behavior, presumably through the activation of neurons involved in descending inhibition (Burkey et al., 1999; Flores et al., 2004). The spinal effect of DA appears to depend on its local concentration. Low levels of DA may activate the anti-nociceptive D2 receptors, whereas higher levels would activate the pro-nociceptive D1 receptors (Paulus and Trenkwalder, 2006).

Interestingly, there appears to be an interaction between the noradrenaline transporter (NAT) and DA release in areas like the VTA, LC and dorsal hippocampus (Moron et al., 2002; Carboni and Silvagni, 2004; Guiard et al., 2008). Similarly, a small population of DA-synthesizing cells exists in DRGs (Millan, 2002) and consequently, it is possible that noradrenergic neurons constitute an important source of DA in the dorsal horn to control pain. Moreover, there is also an interaction between the DA and opioid system, and significantly, dopaminergic neurons are necessary for the anti-nociceptive effect of morphine, particularly in the PAG (Flores et al., 2004; Meyer et al., 2009). These findings suggest that dopaminergic pathways and their regulation by noradrenergic inputs fulfill an important role in the control of nociceptive transmission. In this context, antidepressants that act on the dopaminergic system might represent new therapeutic tools in the treatment of chronic pain, such as bupropion or more recently, triple reuptake inhibitors (TRIs: Xie et al., 2001; Hache et al., 2011; Navratilova et al., 2012).

\section{Histamine in Pain Modulation}

Histamine 2-(4-Imidazolyl)ethylamine) is released from neuronal and non-neuronal sources and it is a mediator of many physiological processes, including the modulation of pain. Histamine is released peripherally in response to tissue injury that directly sensitizes nociceptors and it contributes to the generation of pain hypersensitivity (Kajihara et al., 2010). By contrast, the role of histamine in the CNS remains unclear. Preclinical studies have shown that histamine injected directly into supraspinal areas (e.g., the somatosensory cortex or hippocampus) attenuates pain (Erfanparast et al., 2010; Tamaddonfard and Hamzeh-Gooshchi, 2014), while intrathecal injection of histamine appears to facilitate nociception (Yoshida et al., 2005). Histamine interacts with four receptor subtypes (H1, H2, H3 and H4), which are expressed in both presynaptic and post-synaptic neurons (Brown et al., 2001). H1 and H2 are excitatory receptors mainly located postsynaptically (Brown et al., 2001; Zhang et al., 2013), and systemic inhibition of these receptors has antinociceptive effects in rats when assessed with the formalin test (Mojtahedin, 2016). H3 receptors are predominantly expressed in the central and peripheral nervous system (Panula et al., 2015). This receptor is mainly localized presynaptically in histaminergic neurons with inhibitory activity that regulate the levels of histamine (Hough and Rice, 2011), while its post-synaptic localization in non-histaminergic neurons regulates the release of neurotransmitters, such as ACh, dopamine, 5-HT and noradrenaline (Obara et al., 2019). H4 receptors possess inhibitory activity and they are expressed in neurons, although their location in the nervous system remains uncertain (Connelly et al., 2009). Thus, histamine exerts different effects on pain modulation depending on the receptor subtype with which it interacts and no histaminergic agents have been approved until now for the management of chronic pain.

\section{MONOAMINERGIC DYSFUNCTION IN CHRONIC PAIN}

Chronic pain induces monoaminergic plasticity at peripheral and central areas which lead to the classical signs of persistent pain (Figure 1 and Table 1; Hains et al., 2002; Sounvoravong et al., 2004; Heinricher et al., 2009; Morgado et al., 2011; Alba-Delgado et al., 2013). Thus, a review of the monoaminergic dysfunction in chronic pain may identify potential drug targets.

\section{Noradrenaline}

Peripheral $\alpha$-adrenergic stimulation does not affect pain sensation in healthy conditions but interestingly, such excitation enhances pain sensation in chronic pain (Shea and Perl, 1985). This pro-nociceptive effect is the result of increased excitability of primary sensory neurons, known as hypersensitivity to innocuous and noxious stimuli. The increase in excitability is the result, in part, of neuroplastic changes in animal models of chronic pain, driven possibly by an increase in $\alpha 2$ - and $\alpha 1 B-A R$ mRNA expression in DRGs (Sato and Perl, 1991; McLachlan et al., 1993; Bossut and Perl, 1995; Xie et al., 2001; Maruo et al., 2006) and by upregulation of $\alpha 1$ ARs on cutaneous nociceptive afferents (Drummond et al., 2014). Interestingly, this excitability is enhanced by $\alpha 2$ agonists while it is blocked by administration of $\alpha 2$ antagonists (Sato and Perl, 1991; McLachlan et al., 1993). Based on these studies, it was proposed that the effect of the increase in $\alpha 2$ AR expression on afferents contributes to the maintenance of chronic pain, making this a potential target to relieve peripheral chronic pain.

Spinal $\alpha$-ARs are thought to play an opposite role to peripheral ARs in chronic pain. An increase in spinal $\alpha 2 \mathrm{~A}-$ ARs has been reported in sheep with inflammatory pain (Brandt and Livingston, 1990), while in rats, neuropathic pain has been associated with an increase in spinal $\alpha 2 \mathrm{C}$ ARs but not $\alpha 2 \mathrm{~A}-\mathrm{ARs}$ (Stone et al., 1999). Similarly, the number of spinal $\alpha 2 \mathrm{~A}-\mathrm{ARs}$ in spinal nerve ligation (SNL) rats remains unaltered but surprisingly, an increase in the efficacy of coupling between $\alpha 2 \mathrm{~A}$ and $\mathrm{G} \alpha$ subunits has been reported (Bantel et al., 2005). Additionally, upregulation of the spinal NAT has been reported in such SNL rats (Rojo et al., 2012). These results suggest that chronic pain induces adaptive changes in response to reduced descending noradrenergic tone (Hughes et al., 2013). Interestingly, the increase in $\alpha 2 \mathrm{~A}-\mathrm{AR}$ efficiency could explain why antidepressants induce spinal analgesia in chronic pain conditions (Luo et al., 1994; Malmberg et al., 2001; Bantel et al., 2005). These central adaptive changes suggest that after nerve injury, the descending noradrenergic inhibitory tone may be 


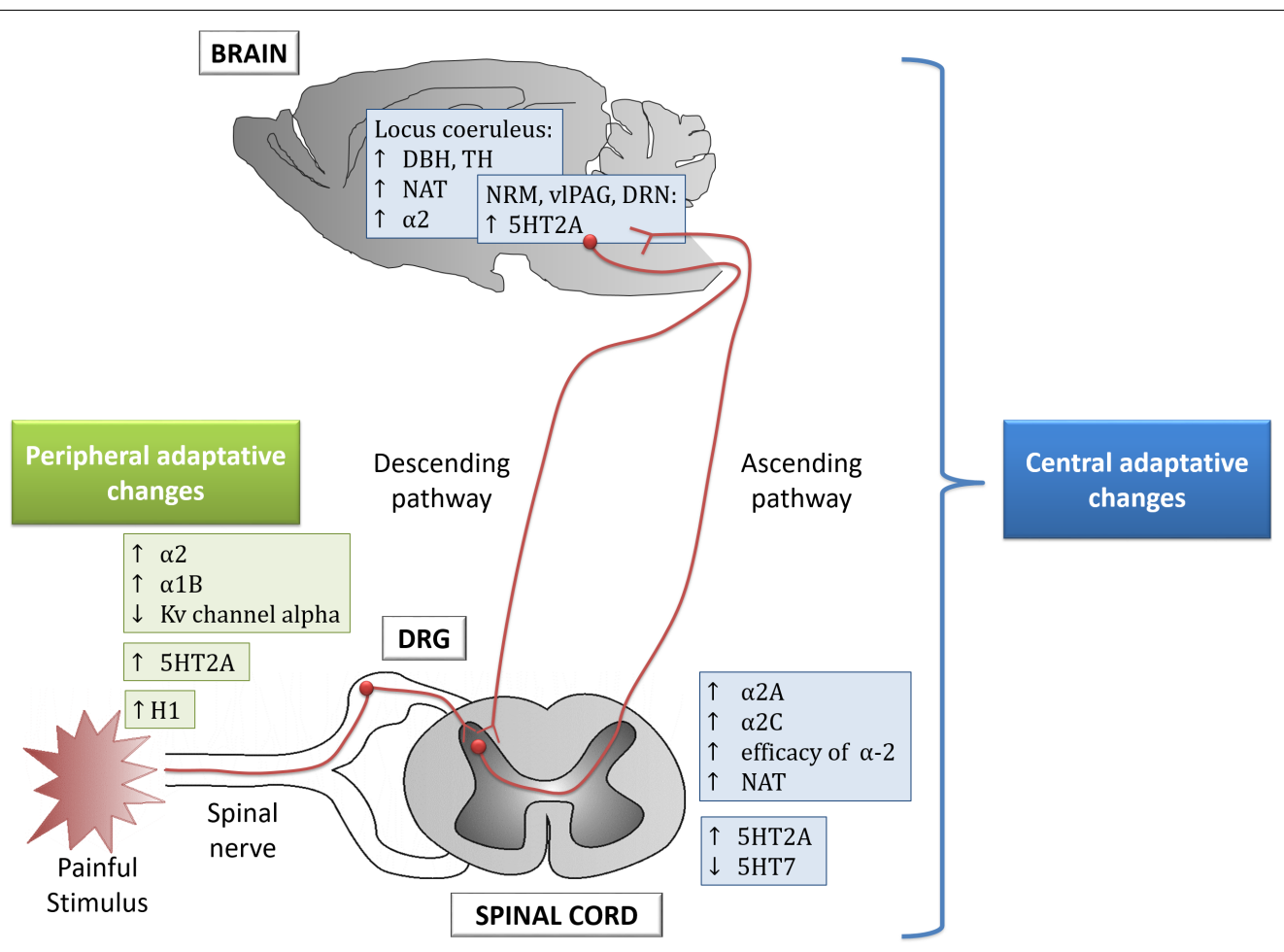

FIGURE 1 | Peripheral and central adaptive changes observed in animal models of chronic pain. DRG, dorsal root ganglion; DRN, dorsal raphe nucleus; RMN, raphe magnus nucleus; vIPAG, ventrolateral periaqueductal gray; DBH, dopamaine beta-hydroxylase; TH, tyrosine hydroxylase; NAT, noradrenaline transporter.

compromised but interestingly, the increase in the $\alpha 2-\mathrm{AR}$ efficacy can be considered beneficial for the effect of NA reuptake inhibitors.

Although spinal adrenergic alterations have received special attention in the development of chronic pain, supraspinal noradrenergic areas have also been seen to change in a manner that is relevant to chronic pain. Thus, an increase in dopamine beta-hydroxylase $(\mathrm{DBH})$, tyrosine hydroxylase (TH), NAT and $\alpha 2$-ARs has been observed in the LC of rats with long-term neuropathic pain (Alba-Delgado et al., 2013; Tazawa et al., 2015). All these changes might try to balance the reduced spinal noradrenergic tone observed in neuropathic pain, although in recent decades these alterations have been seen to be relevant for the development of secondary co-morbidities in chronic pain, such as anxiety and depression (Alba-Delgado et al., 2013, 2018). It has also recently been demonstrated that neuropathic pain induces LC-BLA overactivation, leading to anxiety-like behaviors, and enhancing the aversive learning and memory index (LlorcaTorralba et al., 2019). In addition, optogenetic activation of the LC-PFC (prefrontal cortex) pathway exacerbates spontaneous pain, producing aversion and increasing anxiety-like behavior (Hirschberg et al., 2017). Interestingly, other noradrenergic nuclei (A5 and A7) contribute to the maintenance of chronic pain. Thus, the A7 seems to exert an inhibitory effect on LC neurons in neuropathic pain (Wei and Pertovaara, 2013), while the A5 nucleus sends projections to the dorsal reticular nucleus (DRt) that contributes to the facilitation of pain transmission in the spinal cord via $\alpha 1$-ARs (Lima and Almeida, 2002; Martins et al., 2015).

\section{Serotonin}

The serotonergic descending pain pathways also experiences alterations in animal models of chronic pain. The effect of spinal 5-HT may be either inhibitory or facilitatory, depending on the acute or chronic state of pain (Millan, 2002). Thus, intrathecal administration of 5-HT produces anti-nociception against acute stimuli of diverse nature (Crisp et al., 1991; Bardin et al., 1997), whereas the depletion of 5-HT pathways in models of neuropathic (chronic) pain prevents hypersensitivity (Suzuki et al., 2004; Rahman et al., 2006). Interestingly, reduced spinal 5-HT levels in mice deficient for the 5-HT transporter (5$\mathrm{HTT}^{-/-}$) is associated with decreased thermal hypersensitivity, a symptom caused by peripheral sensitization in pain (Vogel et al., 2003). Thus, it has been suggested that 5-HT might facilitate persistent pain while it has also been proposed that 5-HT could be inhibitory in neuropathic pain.

Regarding 5-HT receptor stimulation, 5-HT1As have been implicated in both facilitatory (i.e., pronociception; Alhaider and Wilcox, 1993) and inhibitory pain (i.e., anti-nociception; Bardin et al., 2001). The activation of 5-HT1A autoreceptors at the supraspinal levels regulates 5 -HT release to the spinal cord and therefore, acute 5-HT1A agonist administration dampens 5-HT release in the spinal cord (Sprouse and Aghajanian, 1987). In animal models of neuropathic pain, the antagonism of 5-HT1A receptors induces analgesia or 
TABLE 1 | Monoaminergic dysfunction in preclinical models of pain.

\begin{tabular}{|c|c|c|c|c|}
\hline System & Finding reported & Model of pain & Animal & References \\
\hline \multicolumn{5}{|l|}{ Noradrenergic } \\
\hline Peripheral & $\uparrow \alpha 2-\mathrm{AR}$ & Sciatic nerve ligation & Rat & McLachlan et al., 1993 \\
\hline \multirow[t]{2}{*}{ (DRG) } & $\uparrow \alpha 1 \mathrm{~B}-\mathrm{AR}$ & SNL, Sciatic nerve ligation & Rat & Xie et al., 2001; Maruo et al., 2006 \\
\hline & $\downarrow$ Kv channel alpha & $\mathrm{CCl}$ & Rat & Kim et al., 2001, 2002 \\
\hline \multirow[t]{4}{*}{ Spinal } & $\uparrow \alpha 2 \mathrm{~A}-\mathrm{AR}$ & Inflammatory pain & Sheep & Brandt and Livingston, 1990 \\
\hline & $\uparrow \alpha 2 \mathrm{C}-\mathrm{AR}$ & $\mathrm{SNL}$ & Rat & Stone et al., 1999 \\
\hline & $\uparrow$ Efficacy of coupling between $\alpha-2 A R$ and $G \alpha$ & SNL & Rat & Bantel et al., 2005 \\
\hline & $\uparrow N A T$ & SNL & Rat & Rojo et al., 2012 \\
\hline Supraspinal & $\uparrow \mathrm{TH}$ & & & \\
\hline \multirow[t]{3}{*}{ (locus coeruleus) } & $\uparrow \mathrm{NAT}$ & $\mathrm{CCl}$ & Rat & Alba-Delgado et al., 2013 \\
\hline & $\uparrow \alpha 2-\mathrm{AR}$ & & & \\
\hline & $\uparrow \mathrm{DBH}$ & SNL & Rat & Tazawa et al., 2015 \\
\hline \multicolumn{5}{|l|}{ Serotonergic } \\
\hline Peripheral & $\uparrow 5-H T 2 A$ in DRG & Vincristine-induced neuropathy & Rat & Thibault et al., 2008 \\
\hline \multirow[t]{4}{*}{ Spinal } & $\uparrow 5$-HT2A in ipsilateral side of dorsal horn & CFA & Rat & Zhang et al., 2001, 2002 \\
\hline & $\uparrow 5-\mathrm{HT} 2 \mathrm{~A}$ in the lumbar dorsal horn & Vincristine-induced neuropathy & Rat & Thibault et al., 2008 \\
\hline & $\downarrow 5$-HT7 in dorsal spinal cord & Formalin test & Rat & Rocha-González et al., 2005 \\
\hline & $\downarrow 5-\mathrm{HT7}$ in ipsilateral dorsal spinal cord & SNL & Rat & Amaya-Castellanos et al., 2011 \\
\hline \multirow[t]{3}{*}{ Supraspinal } & $\uparrow 5$-HT2A bilateral NRM & CFA & Rat & \\
\hline & $\uparrow 5-H T 2 A$ vIPAG & CFA & Rat & Zhang et al., 2001, 2002 \\
\hline & $\uparrow 5-H T 2 A$ DRN & CFA & Rat & \\
\hline \multicolumn{5}{|l|}{ Dopaminergic } \\
\hline \multirow[t]{2}{*}{ Supraspinal } & $\downarrow \mathrm{D} 2$ receptor in the NAc & $\mathrm{SNI}$ & Rat & Sagheddu et al., 2015 \\
\hline & Disruption on D2 and D3 receptors in the hippocampus & $\mathrm{SNI}$ & Rat & Cardoso-Cruz et al., 2014 \\
\hline \multicolumn{5}{|l|}{ Histaminergic } \\
\hline Peripheral & $\uparrow \mathrm{H} 1$ receptor in nociceptive afferent neurons & Capsaicin-induced hyperalgesia & Guinea pig & Kashiba et al., 2001 \\
\hline
\end{tabular}

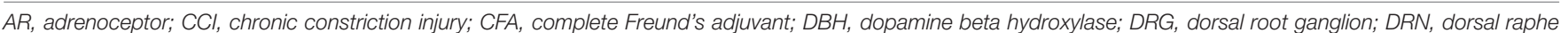

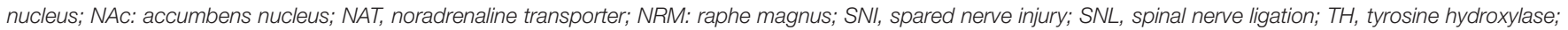
VIPAG, ventrolateral periaqueductal gray.

it potentiates the effect of morphine, tramadol and antidepressants (Rojas-Corrales et al., 2000, 2005; Ardid et al., 2001; Anjaneyulu and Chopra, 2004), suggesting a facilitatory role for this receptor in neuropathic pain at least. By contrast, chronic administration of the 5-HT1A agonist F13640 induced analgesia in an animal model of constriction injury of the infraorbital nerve (Colpaert, 2006; Colpaert et al., 2006). This response could be due to the desensitization of autoreceptors in supraspinal areas following chronic 5-HT1A administration. Therefore, the pro-nociceptive effects of 5HT1A agonist administration have been attributed to presynaptic 5-HT1A, while anti-nociceptive effects could be due to the post-synaptic 5-HT1A activation at the spinal level (Colpaert, 2006).

Antagonism of 5-HT2 and 5-HT3 receptors is thought to dampen analgesia or potentiate the effect of some drugs due to its role in descending facilitation pain (Ali et al., 1996; Green et al., 2000). Interestingly, there are increases in the prominence of these receptors in association with chronic pain. Thus, an increase of 5-HT2A receptor mRNA was evident in DRGs, the
RMN, ventrolateral PAG (vlPAG), dorsal raphe nucleus (DRN) and dorsal horn in a model of inflammatory pain (Zhang et al., 2001, 2002). Similarly, an increase in the 5-HT2A receptor has been reported in the lumbar dorsal horn in association with neuropathic pain (Thibault et al., 2008). Clinical studies reported an increase in 5-HT2A receptor binding in the PFC related to the pain evoked by heat (Kupers et al., 2009), suggesting a clear facilitation role for this receptor. As such, antagonism of this receptor could be a potential target for the treatment of chronic pain.

Although 5-HT3 receptor expression remains unaltered in the dorsal spinal horn in neuropathic pain (Peters et al., 2010), antagonism of the 5-HT3 receptor reduces the second but not the first phase of the formalin test, suggesting that the agonism of the 5-HT3 receptor would facilitate chronic pain (Glaum et al., 1988, 1990; Green et al., 2000). In addition, an increase in serotonergic fibers ( 5 weeks after spinal cord injury -SCI) was proposed to contribute to the maintenance of mechanical allodynia via 5-HT3 receptor activation (Oatway et al., 2004). These findings suggest that 5-HT3 and 5-HT2A receptors that participate in facilitatory 
descending pain contribute to the central sensitization in chronic pain conditions (Bannister and Dickenson, 2017).

There is little information available from a clinical setting, although a decrease in mechanical allodynia was observed in some patients with neuropathic pain treated with a 5HT3 receptor antagonist (McCleane et al., 2003) but not in others (Tuveson et al., 2011). Although selective 5-HT3 receptor antagonists (used as anti-emetics) have inflammatory and analgesic properties in patients suffering inflammatory rheumatic diseases and fibromyalgia (Stratz and Muller, 2000; Vergne-Salle et al., 2011), there are no clinically effective drugs that act on these receptors.

The 5-HT7 receptor has been little studied in chronic pain. While it is thought to be less prominent in the dorsal spinal cord of neuropathic rats, its antagonism seems to produce analgesic properties in neuropathic and inflammatory pain models (Rocha-González et al., 2005; Amaya-Castellanos et al., 2011; Bannister and Dickenson, 2017).

Thus, it is difficult to elucidate the role of 5 -HT and its receptors in pain because such effects are highly dependent on the 5-HT receptor subtype stimulated but also, on the pathological state of the subject.

\section{Dopamine}

Interest in the role of the dopaminergic system in pain has augmented in the last decade. Some changes in the mesocorticolimbic circuit have been reported, considered as a possible target to combat the negative affective states associated with chronic pain. A decrease in D2 receptor expression has been reported in the nucleus accumbens (NAc) of animal models of neuropathic pain, as well as changes in D2 and D3 receptor expression in the hippocampus (Cardoso-Cruz et al., 2014; Sagheddu et al., 2015). In addition, a reorganization of NAc connectivity with cortical areas contralateral (to the injured limb) has been reported in a model of long-term neuropathic pain (Chang et al., 2014). The dopaminergic alterations studied seem to contribute to the negative motivational-affective (Taylor et al., 2003; Scott et al., 2006; Chang et al., 2014) and cognitive aspects of pain (Cardoso-Cruz et al., 2014).

\section{Histamine}

The histaminergic system seems play an important role in the development of hypersensitivity in neuropathic pain (Obara et al., 2019). Histamine is released peripherally by mast cells that are involved in the inflammatory process through the recruitment of macrophages and neutrophils following nerve injury (Calvo and Bennett, 2012). In fact, mast cell depletion prevents mechanical hypersensitivity in a mouse model of persistent pain (Kaur et al., 2017). These mast cells proliferate and can degranulate for up to 2 weeks or even for months under certain conditions of nerve injury (Hayashi et al., 2008). In terms of the histamine receptors, an increase in $\mathrm{H} 1$ receptor expression has been observed in nociceptive afferent neurons after nerve injury (Baron et al., 2001; Kashiba et al., 2001) and interestingly, histamine seems to activate the pruriceptors that induce the release of inflammatory mediators involved in pruritus (itching: Parsons and Ganellin, 2006). There is also evidence indicating that histamine-induced itching can convert into pain associated with neuropathic hypersensitivity (Baron et al., 2001). Thus, the histaminergic system may be subject to alterations that contribute to the maintenance of chronic pain.

\section{ANALGESIC DRUGS WITH MONOAMINERGIC ACTION}

\section{Antidepressants}

The analgesic activity of antidepressants has been widely demonstrated in animals and humans. Although antidepressants can be used to alleviate certain types of chronic pain, mainly neuropathies, their mechanism of action as analgesics remain unclear (Obata, 2017). Given the central role of monoamines in pain modulation it is highly likely that antidepressants exert some effect on pain circuits. The hypotheses regarding the analgesic activity of these drugs focuses on their ability to augment the presence of monoamines by inhibiting NA and 5-HT reuptake. Antidepressants block NA and 5-HT transporters, preventing presynaptic reuptake and thereby increasing the post-synaptic NA and/or 5-HT that reinforces descending pain inhibitory pathways (Hache et al., 2011; Obata, 2017). However, other actions on the monoaminergic system have also been described for these drugs, as described below (Table 2).

\section{Action on the Noradrenergic System Preclinical Studies}

Animal studies suggest that $\alpha 2$-ARs are involved in the analgesia mediated by antidepressants (Ghelardini et al., 2000; Hajhashemi et al., 2014), the most relevant evidence provided by the loss of analgesia induced by amitriptyline in $\alpha 2-\mathrm{AR} \mathrm{KO}$ mice (Ozdogan et al., 2004). In addition to $\alpha 2$-ARs, other adrenergic receptors have also been implicated in the analgesia mediated by antidepressants. Thus, the $\alpha 1$-AR antagonist prazosin blocked the anti-nociceptive effect of nortriptyline, maprotiline, milnacipran, and imipramine in the formalin test (Yokogawa et al., 2002). In terms of $\beta$-ARs, initial studies reported that $\beta 1$ - and $\beta 2$-ARs participated in the analgesia mediated by desipramine (DMI) and nortriptyline, in both thermal and chemical models of acute pain (Mico et al., 1997). Interestingly, in neuropathic mice lacking $\beta 2$-ARs chronic treatment with DMI, nortriptyline and reboxetine failed to produce analgesia (Yalcin et al., 2009), consistent with preclinical findings where repeated stimulation of $\beta 2$-ARs seem to be necessary and sufficient to produce a therapeutic effect (Choucair-Jaafar et al., 2009, 2011; Yalcin et al., 2009, 2010). More recently, mianserin, a tetracyclic antidepressant, was shown to produce analgesia in a model of diabetic neuropathic pain through its interactions with both $\alpha 2$ - and $\beta$-ARs (Ücel et al., 2015). In addition, antidepressants may possibly have peripheral effects, with nortriptyline and venlafaxine mediating the stimulation of $\beta 2$-ARs in non-neuronal cells, thereby inhibiting the production of the cytokine tumor necrosis factor $\alpha(\mathrm{TNF} \alpha)$, provoking an anti-allodynic effect in a rat model of neuropathic pain (Bohren et al., 2013).

All the data available suggest that the analgesic action of antidepressants mainly occurs at the spinal level 
TABLE 2 | Antidepressants with analgesic action via monoaminergic system.

\begin{tabular}{|c|c|c|c|c|c|c|c|c|c|}
\hline & Drugs & Effective Dose & Model of pain & Nociceptive test & Effect & Receptors & $\begin{array}{l}\text { Effect } \\
\text { antagonized by }\end{array}$ & Animal & References \\
\hline \multicolumn{10}{|c|}{ Noradrenaline } \\
\hline \multirow[t]{13}{*}{ Acute pain } & Amitriptyline & 15 mg/Kg i.p. & & Hot plate & $\uparrow$ Latency & $\alpha 2-A R$ & $\begin{array}{l}\text { Reserpine (2 mg/kg } \\
\text { i.p.) }\end{array}$ & Mice & $\begin{array}{l}\text { Ghelardini et al., } \\
2000\end{array}$ \\
\hline & Imipramine & & $\begin{array}{l}\text { Abdominal } \\
\text { constriction }\end{array}$ & Acetic acid test & $\downarrow$ No constriction & & $\begin{array}{l}\text { Yohimbine } \\
\text { (3 mg/kg i.p.) }\end{array}$ & & \\
\hline & & & & & & $\alpha 2 A-A R$ & $\begin{array}{l}\text { BRL } 44408 \\
\text { (1 mg/kg i.p.) }\end{array}$ & & \\
\hline & (+) Oxaprotiline & 10, 35 mg/kg i.p. & $\begin{array}{l}\text { Abdominal } \\
\text { constriction }\end{array}$ & Acetic acid test & $\downarrow$ No constriction & $\alpha 2-A R$ & $\begin{array}{l}\text { RX821002 } \\
\text { (1 mg/kg s.c.) }\end{array}$ & Mice & Gray et al., 1999 \\
\hline & Paroxetine & 10 mg/kg i.p. & & & & & & & \\
\hline & Sibutramine & 5, 25 mg/kg i.p. & & & & & & & \\
\hline & Dothiepine & 5, 30 mg/kg i.p. & & & & & & & \\
\hline & Amitriptyline & 5, 25 mg/kg i.p. & & & & & & & \\
\hline & TCA: Amitriptyline & 10 mg/kg i.p. & Heat stimulation & Tail-flick test & $\begin{array}{l}\text { Not displays } \\
\text { analgesic effect }\end{array}$ & $\alpha 2-A R$ & & $\alpha 2-A R$ KO Mice & $\begin{array}{l}\text { Ozdogan et al., } \\
2004\end{array}$ \\
\hline & Desipramine & $\begin{array}{l}4,20,40 \mathrm{mg} / \mathrm{kg} \\
\text { i.p. }\end{array}$ & & Hot plate test & $\uparrow$ Latency & $\beta 1-\mathrm{AR}$ & $\begin{array}{l}\text { CGP20712A } \\
(1 \mathrm{mg} / \mathrm{kg} \text { s.c.) }\end{array}$ & Mice & Mico et al., 1997 \\
\hline & Nortriptyline & $\begin{array}{l}2,4,20 \\
40 \text { mg/kg i.p. }\end{array}$ & Heat stimulation & Tail flick test & $\uparrow$ Latency & $\beta 2-A R$ & $\begin{array}{l}\text { ICl118551 } \\
(30 \mu \mathrm{g} / \mathrm{kg} \text { s.c. })\end{array}$ & & \\
\hline & & & $\begin{array}{l}\text { Abdominal } \\
\text { constriction }\end{array}$ & Acetic acid test & $\downarrow$ No constriction & & & & \\
\hline & & & Inflammatory & $\begin{array}{l}\text { Formalin test (first } \\
\text { phase) }\end{array}$ & $\downarrow$ licking time & & & & \\
\hline \multirow[t]{12}{*}{ Chronic pain } & Venlafaxine & 40 mg/kg i.p. & Neuropathic: CCl & Plantar test & $\uparrow$ Latency & $\alpha 2-A R$ & $\begin{array}{l}\text { Yohimbine } \\
\text { (5 mg/kg i.p.) }\end{array}$ & Rat & $\begin{array}{l}\text { Hajhashemi et al., } \\
2014\end{array}$ \\
\hline & & $\begin{array}{l}\text { 10, } 20 \text { mg/kg i.p. } \\
\text { (14 days) }\end{array}$ & & von Frey test & $\begin{array}{l}\uparrow \text { Withdrawal } \\
\text { threshold }\end{array}$ & & & & \\
\hline & SNRI: Duloxetine & $\begin{array}{l}10 \text { mg/kg s.c. } \\
\text { (3 days) }\end{array}$ & Neuropathic: SNL & von Frey test & $\begin{array}{l}\uparrow \text { Withdrawal } \\
\text { threshold }\end{array}$ & $\alpha 2-A R$ & $\begin{array}{l}\text { Idazoxan } \\
(30 \mu \mathrm{g} / 20 \mu \mathrm{g} \text { i.t.) }\end{array}$ & Rat & Ito et al., 2018 \\
\hline & $\begin{array}{l}\text { Nortriptyline, } \\
\text { Imipramine }\end{array}$ & 5 mg/kg i.p. & Inflammatory & $\begin{array}{l}\text { Formalin test (late } \\
\text { phase) }\end{array}$ & $\downarrow$ Licking time & $\alpha 1-A R$ & $\begin{array}{l}\text { Prazosin (1 mg/ } / \mathrm{kg} \\
\text { i.p.; } 5 \mu \text { g, i.c.v. })\end{array}$ & Rat & $\begin{array}{l}\text { Yokogawa et al., } \\
2002\end{array}$ \\
\hline & Nisoxetine & 2.5 mg/kg i.p. & & & & & & & \\
\hline & Maprotiline & 10 mg/kg i.p. & & & & & & & \\
\hline & Milnacipran & 5 mg/kg i.p. & & & & & & & \\
\hline & Fluvoxamine & 20 mg/kg i.p. & & & & & & & \\
\hline & Mianserin & $\begin{array}{l}30,45 \mathrm{mg} / \mathrm{kg} \\
\text { o.p. (14 days) }\end{array}$ & Neuropathic: STZ & Randall-Selitto & $\begin{array}{l}\uparrow \text { Withdrawal } \\
\text { threshold }\end{array}$ & $\alpha 2-A R$ & $\begin{array}{l}\text { Phentolamine } \\
\text { ( } 5 \text { mg/kg o.p.; } \\
14 \text { days) }\end{array}$ & Rat & Ücel et al., 2015 \\
\hline & & & & Von Frey & $\begin{array}{l}\uparrow \text { Withdrawal } \\
\text { threshold }\end{array}$ & $\beta-A R$ & $\begin{array}{l}\text { Propranolol } \\
\text { (5 mg/kg o.p. } \\
14 \text { days) }\end{array}$ & & \\
\hline & & & & Plantar test & $\uparrow$ Latency & & & & \\
\hline & & & & Hot plate test & $\uparrow$ Latency & & & & \\
\hline
\end{tabular}




\begin{tabular}{|c|c|c|c|c|c|c|c|c|c|}
\hline & Drugs & Effective Dose & Model of pain & Nociceptive test & Effect & Receptors & $\begin{array}{l}\text { Effect } \\
\text { antagonized by }\end{array}$ & Animal & References \\
\hline & Nortriptyline & $\begin{array}{l}5 \text { mg/kg i.p. } \\
\text { (2-3 weeks) }\end{array}$ & Neuropathic: STZ & von Frey test & $\begin{array}{l}\uparrow \text { Withdrawal } \\
\text { threshold }\end{array}$ & $\beta 2-A R$ & $\begin{array}{l}\text { ICl118551 } \\
\text { (2 mg/kg i.p.) }\end{array}$ & Mice & $\begin{array}{l}\text { Choucair-Jaafar } \\
\text { et al., } 2009\end{array}$ \\
\hline & Desipramine & $\begin{array}{l}5 \mathrm{mg} / \mathrm{kg} \text { i.p. } \\
\text { (4 weeks) }\end{array}$ & Neuropathic: SNC & von Frey test & $\begin{array}{l}\uparrow \text { Withdrawal } \\
\text { threshold }\end{array}$ & $\beta 2-A R$ & $\begin{array}{l}\text { ICl118551 } \\
\text { (2 mg/kg i.p.; } \\
3 \text { days) }\end{array}$ & $\beta 2-A R+/+$ Mice & Yalcin et al., 2009 \\
\hline & Venlafaxine & $\begin{array}{l}10 \text { mg/kg i.p. } \\
\text { (4 weeks) }\end{array}$ & & & & & & $\begin{array}{l}\beta 2-\mathrm{AR}-/-\mathrm{KO} \\
\text { Mice }\end{array}$ & \\
\hline & Reboxetine & $\begin{array}{l}0.8 \mathrm{mg} / \mathrm{kg} \text { i.p. } \\
\text { ( } 4 \text { weeks) }\end{array}$ & & & & & & & \\
\hline & Nortriptyline & $\begin{array}{l}5 \text { mg/kg i.p. } \\
\text { (2-3 weeks) }\end{array}$ & Neuropathic: SNC & von Frey test & $\begin{array}{l}\text { Not displays } \\
\text { analgesic effect }\end{array}$ & $\beta 2-A R$ & $\begin{array}{l}\text { Terbutaline } \\
\text { (0.5 mg/kg i.p.) }\end{array}$ & B2AR -/- Mice & Bohren et al., 2013 \\
\hline & Venlafaxine & & & & $\begin{array}{l}\uparrow \text { Withdrawal } \\
\text { threshold }\end{array}$ & & & C57BL/6J Mice & \\
\hline \multicolumn{10}{|l|}{ Serotonin } \\
\hline \multirow[t]{5}{*}{ Acute pain } & Venlafaxine & 80 mg/kg i.p. & & Hot plate test & $\uparrow$ Latency & $5-H T 1 A$ & $\begin{array}{l}\text { 8-OH-DPAT } \\
\text { (0.062 mg/kg s.c.) }\end{array}$ & Mice & $\begin{array}{l}\text { Berrocoso and } \\
\text { Mico, } 2007\end{array}$ \\
\hline & Fluoxetine & $\begin{array}{l}10 \text { mg/kg i.p. } \\
\text { (7 days) }\end{array}$ & $\begin{array}{l}\text { Abdominal } \\
\text { constriction }\end{array}$ & Acetic acid test & $\downarrow$ No constriction & $5-\mathrm{HT} 1 \mathrm{~A}$ & $\begin{array}{l}\text { Pindolol (10 mg/kg } \\
\text { i.p.) }\end{array}$ & Mice & Singh et al., 2001 \\
\hline & Fluoxetine & 0.3-1 nmol/paw & Inflammatory & $\begin{array}{l}\text { Formalin test (first } \\
\text { phase) }\end{array}$ & $\uparrow$ Fliching & & & Rat & $\begin{array}{l}\text { Cervantes-Durán } \\
\text { et al., } 2013\end{array}$ \\
\hline & Paroxetine & $\begin{array}{l}\text { 5, } 10,20 \mathrm{mg} / \mathrm{kg} \\
\text { i.p. }\end{array}$ & $\begin{array}{l}\text { Abdominal } \\
\text { constriction }\end{array}$ & Acetic acid test & $\downarrow$ No constriction & 5-НТ3 & $\begin{array}{l}\text { Ondansetron } \\
(0.1 \mathrm{mg} / \mathrm{kg})\end{array}$ & Mice & Kesim et al., 2005 \\
\hline & Fluvoxamine & 30 mg/kg i.p. & & Paw pressure test & $\uparrow$ Latency & 5-HТ3 & $\begin{array}{l}\text { Granisetron } \\
(1 \mathrm{mg} / \mathrm{kg}, \mathrm{s.c})\end{array}$ & Mice & Honda et al., 2006 \\
\hline \multirow[t]{9}{*}{ Chronic pain } & Fluvoxamine & $\begin{array}{l}\text { 10, } 30 \text { mg/kg i.p; } \\
100 \text { mg i.t. }\end{array}$ & $\begin{array}{l}\text { Neuropathic: Partial } \\
\text { sciatic nerve injury }\end{array}$ & von Frey test & $\begin{array}{l}\uparrow \text { Withdrawal } \\
\text { threshold }\end{array}$ & 5-HT2A/AC & $\begin{array}{l}\text { Ketanserin } \\
\text { (3 mg/kg i.p.; } \\
10 \mathrm{mg} \text { i.t.) }\end{array}$ & Mice & Honda et al., 2006 \\
\hline & Fluoxetine & 0.3-1 nmol/paw & & $\begin{array}{l}\text { Formalin test (late } \\
\text { phase) }\end{array}$ & $\uparrow$ Fliching & 5-HT2A & $\begin{array}{l}\text { Ketanserin } \\
\text { (10 pmol/paw) }\end{array}$ & Rat & $\begin{array}{l}\text { Cervantes-Durán } \\
\text { et al., } 2013\end{array}$ \\
\hline & & & & & & 5-HT2B & $\begin{array}{l}\text { RS- } 127445 \\
(10 \mathrm{pmol} / \mathrm{paw})\end{array}$ & & \\
\hline & & & & & & 5-HT2C & $\begin{array}{l}\text { RS-102221 } \\
\text { (10 pmol/paw) }\end{array}$ & & \\
\hline & & & & & & 5-HТ3 & $\begin{array}{l}\text { Ondansetron (10 } \\
\mathrm{nmol} / \mathrm{paw} \text { ) }\end{array}$ & & \\
\hline & & & & & & $5-\mathrm{HT} 4$ & $\begin{array}{l}\text { GR-113808 (100 } \\
\mathrm{fmol} / \mathrm{paw})\end{array}$ & & \\
\hline & & & & & & 5-HT6 & $\begin{array}{l}\text { SB-258585 } \\
\text { (10 pmol/paw) }\end{array}$ & & \\
\hline & & & & & & 5-HT7 & $\begin{array}{l}\text { SB-269970 (1 } \\
\text { nmol/paw) }\end{array}$ & & \\
\hline & & $\begin{array}{l}3 \mathrm{nmol} / \mathrm{paw} \text { and } \\
\text { i.t. }\end{array}$ & & & $\downarrow$ Fliching & $5-\mathrm{HT} 1 \mathrm{~A}$ & $\begin{array}{l}\text { WAY-100635 (1 } \\
\text { nmol i.t.) }\end{array}$ & & \\
\hline
\end{tabular}


TABLE 2 | Continued

\begin{tabular}{|c|c|c|c|c|c|c|c|c|c|}
\hline & Drugs & Effective Dose & Model of pain & Nociceptive test & Effect & Receptors & $\begin{array}{l}\text { Effect } \\
\text { antagonized by }\end{array}$ & Animal & References \\
\hline & & & & & & 5-HT1B/1D & GR-127935 (1 nmol i.t.) & & \\
\hline & & & & & & $\mathrm{HT} 1 \mathrm{~B}$ & SB-224289 (1 nmol i.t.) & & \\
\hline & & & & & & $5-\mathrm{HT} 1 \mathrm{D}$ & BRL-15572 (1 nmol i.t.) & & \\
\hline & Imipramine & 10 mg/kg i.p. & Inflammatory & $\begin{array}{l}\text { Formalin test (late } \\
\text { phase) }\end{array}$ & $\downarrow$ Licking time & 5-HT3 & $\begin{array}{l}\text { Ondansetron (1 mg/kg } \\
\text { i.p.) }\end{array}$ & Rat & $\begin{array}{l}\text { Yokogawa et al., } \\
2002\end{array}$ \\
\hline & Nortriptyline & 5, 10 mg/kg i.p. & & & & $5-\mathrm{HT} 2$ & $\begin{array}{l}\text { Ketanserin (1, } 2 \text { mg/kg } \\
\text { i.p; } 60 \mu \text { i.c.v.) }\end{array}$ & & \\
\hline & Nisoxetine & 2.5 mg/kg i.p. & & & & & & & \\
\hline & Maprotiline & 10 mg/kg i.p. & & & & & & & \\
\hline & Milnacipran & 5 mg/kg i.p. & & & & & & & \\
\hline & Fluvoxamine & 20 mg/kg i.p. & & & & & & & \\
\hline & Imipramine & 2.5 mg/kg i.p. & & $\begin{array}{l}\text { Formalin test (late } \\
\text { phase) }\end{array}$ & $\uparrow$ Licking time & $5-\mathrm{HT} 4$ & $\begin{array}{l}\text { SDZ-205, } 557 \\
\text { (0.1 mg/kg i.p.) }\end{array}$ & & \\
\hline & Fluoxetine & $\begin{array}{l}10 \mathrm{mg} / \mathrm{kg} \text { i.p. } \\
(3 \text { weeks) }\end{array}$ & Neuropathic: STZ & Randall-Selitto & $\begin{array}{l}\uparrow \text { The vocalization } \\
\text { thresholds }\end{array}$ & $5-\mathrm{HT} 2 \mathrm{~A}$ & $\begin{array}{l}\text { TAT-2ASCV peptide } \\
\text { (30 ng/rat i.t.) }\end{array}$ & Rat & Pichon et al., 2010 \\
\hline & & & & Hot plate & $\uparrow$ Latency & & & & \\
\hline & Fluoxetine & 10, 20 mg/kg i.p. & Neuropathic: STZ & Tail inmersion & $\uparrow$ Latency & $5-\mathrm{HT} 2 \mathrm{~A} / 2 \mathrm{C}$ & $\begin{array}{l}\text { Ritanserin (1, } 2 \text { mg/kg } \\
\text { i.p.) }\end{array}$ & Mice & $\begin{array}{l}\text { Anjaneyulu and } \\
\text { Chopra, } 2004\end{array}$ \\
\hline & & & & Hot-plate test & $\uparrow$ Latency & & & & \\
\hline & Clomipramine & $6 \mathrm{mg} / \mathrm{kg}$ i.v. & $\begin{array}{l}\text { Neuropathic: CCl, } \\
\text { STZ }\end{array}$ & Randall-Selitto & $\begin{array}{l}\uparrow \text { Vocalization } \\
\text { thresholds }\end{array}$ & $5-\mathrm{HT} 1 \mathrm{~A}$ & $\begin{array}{l}\text { WAY 100, } 635 \text { (0.5, } \\
8 \text { mg/kg s.c.) }\end{array}$ & Rat & Ardid et al., 2001 \\
\hline & Venlafaxine & $\begin{array}{l}5,10,20 \mathrm{mg} / \mathrm{kg} \\
\text { i.v. }\end{array}$ & Neuropathic: CCI & Randall-Selitto & $\begin{array}{l}\uparrow \text { Vocalization } \\
\text { thresholds }\end{array}$ & $5-\mathrm{HT} 1 \mathrm{~A}$ & $\begin{array}{l}\text { OH-DPAT (0.5 mg/kg } \\
\text { s.c.) }\end{array}$ & Rat & $\begin{array}{l}\text { Hernandez et al., } \\
2004\end{array}$ \\
\hline & & & & & & & $\begin{array}{l}\text { Antisense } \\
\text { oligodeoxynucleotide } \\
(0.1 \mathrm{nmol} / \mu \mathrm{l})\end{array}$ & & \\
\hline \multicolumn{10}{|l|}{ Dopamine } \\
\hline \multirow[t]{2}{*}{ Acute pain } & Nomifensine & $\begin{array}{l}1.25,2.5,5 \\
10 \mathrm{mg} / \mathrm{kg} \mathrm{s} . \mathrm{c}\end{array}$ & & Tail inmersion & $\begin{array}{l}\text { Not displays } \\
\text { analgesic effect }\end{array}$ & D2 & $\begin{array}{l}\text { Eticlopride } \\
\text { (181.3-270 mg/kg ip) }\end{array}$ & Rat & $\begin{array}{l}\text { Gilbert and } \\
\text { Franklin, } 2001\end{array}$ \\
\hline & & & & Hot plate & $\begin{array}{l}\text { Not displays } \\
\text { analgesic effect }\end{array}$ & & & & \\
\hline \multirow[t]{4}{*}{ Chronic pain } & Amitriptyline & 3-30 mg/kg i.p. & Neuropathic: SNL & $\begin{array}{l}\text { Formalin test } \\
\text { von Frey test }\end{array}$ & $\begin{array}{l}\downarrow \text { Licking time } \\
\uparrow \text { Withdrawal } \\
\text { threshold }\end{array}$ & D2 & Sulpiride (30 $\mu$ g i.t.) & Rat & Chen et al., 2017 \\
\hline & Milnacipran & & & & & & & & \\
\hline & Duloxetine & & & & & & & & \\
\hline & Fluoxetine & & & & & & & & \\
\hline
\end{tabular}

AR, adrenoceptor; CCl, chronic constriction injury; i.p., intraperitoneal injection; i.v, intravenous injection; i.t. intrathecal injection; s.c., subcutaneous injection; SNC: sciatic nerve cuffing; SNL, spinal nerve ligation; STZ streptozotocin-induced diabetic; o.p., oral administration 
(Mico et al., 2006; Ito et al., 2018; Kremer et al., 2018). Indeed, it was recently verified that daily subcutaneous injections of duloxetine attenuate SNL hypersensitivity in rats through NA accumulation in the spinal cord (Ito et al., 2018). Given the role of the LC in analgesia through the descending pain pathway, the effect of antidepressants at this level has been evaluated. As expected, electrophysiological assays have demonstrated the action of antidepressants on the LC neurons, for example the acute administration of venlafaxine (a SNRI) completely inhibits the LC activity modulated by $\alpha 2$-ARs and 5 -HT1A receptors (Berrocoso and Mico, 2007). Interestingly, DMI and duloxetine restored the functional changes in rat LC neurons affected by neuropathic pain, an event that correlated with analgesic behavior (Alba-Delgado et al., 2012). Similarly, functional Magnetic Resonance Imaging (fMRI) showed that chronic administration of DMI ( 14 days, daily i.p. of $10 \mathrm{mg} / \mathrm{kg}$ ) produced greater activation of areas altered by chronic pain in a rat model of neuropathic pain: primary somatosensory cortex, insular cortex, medial globus pallidus, etc. (Jones et al., 2009).

\section{Clinical Approach}

Antidepressants that mainly inhibit noradrenaline reuptake, like DMI and maprotiline, are not a good choice for the treatment of human neuropathic pain (Max et al., 1991, 1992; Vrethem et al., 1997), yet they are used in patients who have not obtained pain relief with other treatments. Clinical evidence suggests that dual antidepressants provide better analgesic efficacy in chronic neuropathic pain. In this regard, the analgesic efficacy of amitriptyline (a tricyclic antidepressant -TCA) is the bestdocumented in complex regional pain syndrome, neuropathic and musculoskeletal pain, and fibromyalgia (Wiffen et al., 2013; Moore et al., 2015; Brown et al., 2016; van den Driest et al., 2017). However, due to the side effects of TCAs, the SNRI duloxetine has been the first choice treatment for peripheral diabetic neuropathic pain and fibromyalgia, with a good safety profile. Despite the efficacy revealed in these conditions, this class of antidepressants is not useful in relieving SCI, phantom limb and HIV neuropathy (Kieburtz et al., 1998; Cardenas et al., 2002; Robinson et al., 2004), yet there is insufficient evidence to prescribe antidepressants for inflammatory chronic pain (Perrot et al., 2006; Richards et al., 2011). Thus, more clinical trials are needed in this area.

Regarding the mechanisms of action, it is believed that the increase of monoamines associated with the inhibition of NA and 5-HT reuptake reinforces inhibitory descending pain pathways (Obata, 2017). Clinical studies have reported the importance of stimulating $\alpha 2 \mathrm{~A}-\mathrm{AR}$ to induce analgesia and indeed, $\alpha 2$ agonist administration has anti-nociceptive activity alone or in combination with opioids (Eisenach et al., 1994, 1995). In clinical practice, antidepressants exhibit little or only intermediate efficacy against acute nociceptive stimuli, while they induce significant analgesia in chronic pain (Poree et al., 1998; Sawynok et al., 2001; Paqueron et al., 2003; Mico et al., 2006). Interestingly, enhanced $\alpha 2 \mathrm{~A}-\mathrm{AR}$ efficiency could explain why antidepressants induce spinal analgesia in chronic pain (Luo et al., 1994; Malmberg et al., 2001; Bantel et al., 2005). Accordingly, drugs acting through $\alpha 2$-ARs such as clonidine is effective in patients with neuropathic pain at doses that are inactive in post-operative pain (Eisenach et al., 1995). Although preclinical evidence indicates that $\beta 2$-ARs are necessary to produce a therapeutic effect (Choucair-Jaafar et al., 2009, 2011; Yalcin et al., 2009, 2010), there is one clinical report supporting the efficacy of $\beta 2$-ARs in combating neuropathic pain (Cok et al., 2010).

\section{Action on the Serotonergic System Preclinical Studies}

Like noradrenaline, the main putative analgesic mechanism for $5-\mathrm{HT}$ is the increase of 5 -HT neurotransmission by 5 -HT receptor stimulation and the ensuing modulation of descending pain inhibition. However, specific 5-HT receptor subtypes can dampen or enhance neuronal spinal cord activity (Millan, 2002), thereby weakening or potentiating descending pain transmission. Therefore, the role of 5-HT in analgesia remains controversial.

The effect of selectively inhibiting 5-HT reuptake has mainly been studied using SSRIs like fluoxetine. The anti-nociceptive effect of fluoxetine has been demonstrated in several animal models of acute and chronic pain (tail flick, hot plate and streptozotocin diabetic mice), although the data obtained have at times been contradictory (Singh et al., 2001; Cervantes-Durán et al., 2013). Thus, 5-HT has a pro-nociceptive peripheral effect, as reflected by the enhanced pain in the formalin test after local fluoxetine injection. However, systemic and intrathecal injection reduces any pro-nociceptive behavior in this test (CervantesDurán et al., 2013). The peripheral pro-nociceptive effect seems to be mediated by the activation of $5-\mathrm{HT} 2 \mathrm{~A} / 2 \mathrm{~B} / 2 \mathrm{C} / 3 / 4 / 6 / 7$ receptors, while systemic and intrathecal anti-nociception seems to be mediated by $5-\mathrm{HT} 1 \mathrm{~A} / 1 \mathrm{~B} / 1 \mathrm{D} / 5 \mathrm{~A}$ receptor activation.

The pro-nociceptive effect of 5-HT on some 5-HT receptors reveals the importance of co-administering certain 5-HT antagonists in pain management. As such, analgesia is enhanced when 5-HT1A receptors are blocked with drugs that augment 5-HT. Accordingly, the anti-nociceptive effect of the SNRI venlafaxine (injected subcutaneously) was potentiated in the hot plate test by 5 -HT1A receptors antagonism. Similarly, systemic co-administration of a 5-HT1A receptor antagonist enhanced fluoxetine-induced anti-nociception in the acetic acid test (Singh et al., 2001). Moreover, several studies showed that systemic blockage of 5-HT1A receptors enhances the analgesic effect of SSRIs like fluoxetine (Anjaneyulu and Chopra, 2004) and clomipramine (Ardid et al., 2001) in chronic pain models. In fact, chronic intracerebroventricular (ICV) administration of an antisense oligodeoxynucleotide that silences 5-HT1A receptor synthesis decreases mechanical hypersensitivity of neuropathic rats (Hernandez et al., 2004). These results provide strong evidence of the importance of co-administering selective 5HT1A antagonists to potentiate the anti-nociceptive efficacy of antidepressants.

The antagonism of 5-HT2 receptors potentiates the antinociceptive effect of paroxetine, effect that is blocked by 5 -HT3 antagonist in the acetic acid test (Kesim et al., 2005). However, ketanserin (a 5-HT2A/AC receptor antagonist) antagonizes the anti-allodynic effect of fluvoxamine in a mice model of neuropathic pain. These findings suggest that SSRIs would have 
an opposite effect on $5-\mathrm{HT} 2 \mathrm{~A} / 2 \mathrm{C}$ receptors in acute or chronic pain (Honda et al., 2006).

There is little information about the role of 5-HT receptors in chronic pain and despite the influence of 5-HT on pain modulation, some preclinical studies have reported that the analgesia produced by SSRIs is less potent in chronic than in acute pain models (Bardin et al., 2000; Pichon et al., 2010). In fact, the stimulation of 5-HT2A receptors induces analgesia in healthy but not diabetic neuropathic rats. Thus, the alterations to 5-HT2A receptors that occurs in neuropathic pain is thought to be one of the causes of this inefficacy. In fact, pharmacological disruption of the association between spinal serotonin type 2A (5-HT2A) receptors and their associated PDZ proteins suppresses the analgesia induced by fluoxetine in diabetic and SNL rats (Pichon et al., 2010). Hence, 5-HT2A receptors and PDZ protein interactions might influence the resistance to SSRIinduced analgesia in the management of chronic neuropathic pain. Coupling the pro-nociceptive stimulation of some 5-HT receptors to the 5-HT alterations observed in chronic pain possibly explains the inefficacy of SSRIs in the management of chronic pain.

\section{Clinical Approach}

Although modulation of the 5-HT system could dampen or enhance the magnitude of pain, antidepressants like SSRIs that selectively acting through 5 - $\mathrm{HT}$ are thought to be relatively poor in combating chronic pain (Johansson and Von Knorring, 1979; Anderberg et al., 2000) and therefore, they are not recommended as a first-line therapy for chronic pain. Fluoxetine and paroxetine have been used unsuccessfully to manage non-neuropathic chronic pain like headache and migraine (Saper et al., 1994, 1995), and paroxetine and citalopram have minimal effects on patients suffering painful diabetic neuropathy. Similarly, fluoxetine had no effect in patients with painful diabetic neuropathy (Sindrup et al., 1990, 1992; Max et al., 1992). Animal studies have helped us to elucidate some possible mechanisms responsible for the ineffectiveness of SSRIs in chronic pain, such as the facilitatory role of serotonin on 5-HT3 receptors and the alterations to 5-HT2A receptors in this condition. However, other drugs with dual action on NA and 5-HT reuptake inhibitors have proved to be efficacious in treating several forms of chronic pain, such as venlafaxine and duloxetine for fibromyalgia, migraine and diabetic neuropathy (Kaur et al., 2011; King et al., 2015; Burch, 2019). Hence, further studies should be carried out to assess how to achieve effective analgesia through the 5-HT system. By contrast, there is no evidence to support the use of SSRIs to combat inflammatory chronic pain like rheumatoid arthritis or osteoarthritis (Perrot et al., 2008).

\section{Action on the Dopaminergic System Preclinical Studies}

The descending release of DA in the spinal cord plays an important role in pain modulation, although there are limited studies into the dopaminergic influence on the analgesic effect of antidepressants. Intrathecal injection of bupropion produces a dose-dependent anti-hyperalgesic effect in neuropathic rats, coincident with an increase in $\mathrm{NA}$ and $\mathrm{DA}$ in the spinal cord (Hoshino et al., 2015). In addition, the norepinephrinedopamine reuptake inhibitor nomifensine has a similar potency and efficacy to morphine in the formalin test (Gilbert and Franklin, 2001). It was recently reported that spinal DA receptors are implicated in the anti-hyperalgesic effect of antidepressants in a model of spinal nerve ligation. Pretreatment with a dopamine D2 receptor antagonist abolished the antihyperalgesic effects of intraperitoneal amitriptyline, duloxetine, milnacipran and fluoxetine administration (Chen et al., 2017), suggesting that DA would exert a role in the spinal inhibitory effect of antidepressants. Nevertheless, further studies into the implication of the DA system in pain should be carried out.

\section{Clinical Approach}

Few studies have evaluated the analgesic effectiveness of specific DA reuptake inhibition in chronic pain patients. In this regard, the atypical antidepressant bupropion (a dual noradrenaline and dopamine reuptake inhibitor) decreases the intensity of pain and improves the quality of life of neuropathic pain patients (Semenchuk and Davis, 2000). However, the side effects of this drug are an important drawback as bupropion may be an excessively strong stimulant, inhibiting the appetite. As such, it is absolutely contraindicated in patients with a history of seizures or eating disorders.

Nonetheless, new TRIs have been designed that block all three monoamines by acting on their transporters (DAT, NAT and SERT: dopamine, noradrenaline, and serotonin transporters: Hache et al., 2011). These drugs should theoretically be very efficacious in treating chronic pain and they could be useful when pain is co-morbid with other conditions in which the availability of some monoamines is compromised, such as in depression or Parkinson's disease. However, whether these drugs truly represent an interesting new strategy remains to be fully explored.

\section{Gabapentinoids \\ Preclinical Studies}

Gabapentin was introduced as an antiepileptic drug in 1993 and it was subsequently recognized as a first-line drug for several types of chronic pain. Gabapentinoids have been widely used to treat neuropathic pain and they are mainly characterized by their action on $\alpha 2 \delta$ subunits in primary afferents. However, they may also act on supraspinal areas and stimulate descending pain inhibition, with their supraspinal effects mediated by spinal $\alpha 2$-ARs (Table 3; Takasu et al., 2006). Accordingly, high doses of gabapentin and pregabalin (ICV), and i.v. administration of gabapentin, enhances NA turnover in the spinal cord (Takeuchi et al., 2007; Hayashida et al., 2008; Tanabe et al., 2008), provoking spinal $\alpha 2-\mathrm{AR}$ mediated analgesia. In murine models of neuropathic pain, $\alpha 2$ antagonists suppress the anti-hypersensitivity effect of gabapentinoids following ICV administration (Tanabe et al., 2008). There have been few studies into gabapentinoids and the serotonergic system, yet the increase in 5-HT3 receptors after injury determines the analgesic actions of gabapentinoids (Bee and Dickenson, 2008). Conversely, pregabalin induced pain relief via the intra-accumbens nucleus induces DA release during early but not late neuropathic pain (Kato et al., 2016). Hence, pain chronification leads to 
TABLE 3 | Analgesic action of gabapentinoids, opioids, NSAID's and histaminergic drugs via monoaminergic system.

\begin{tabular}{|c|c|c|c|c|c|c|c|c|}
\hline Drugs & Effective Dose & Model of pain & $\begin{array}{l}\text { Nociceptive } \\
\text { test }\end{array}$ & Effect & Receptors & Effect antagonized by & Animal & References \\
\hline \multicolumn{9}{|l|}{ Gabapentinoids } \\
\hline \multirow[t]{2}{*}{ Gabapentin } & $100 \mu \mathrm{g}$ i.c.v. & $\begin{array}{l}\text { Neuropathic: } \\
\text { PSL }\end{array}$ & Plantar test & $\uparrow$ Latency & $\alpha 2-A R$ & Yohimbine (3 $\mu$ g, i.t.) & Mice & Takasu et al., 2006 \\
\hline & & & von Frey test & $\uparrow$ Latency & & Idazoxan (3 $\mu$ g, i.t.) & & \\
\hline Gabapentin & $1 \mu \mathrm{g}$ intra LC & $\begin{array}{l}\text { Neuropathic: } \\
\text { SNL }\end{array}$ & Randall-Selitto & $\uparrow$ Latency & $\alpha 2-A R$ & Idazoxan (30 ㅆg, i.t.) & Rat & Hayashida et al., 2008 \\
\hline Gabapentin & $100 \mu \mathrm{g}$ i.c.v. & $\begin{array}{l}\text { Neuropathic: } \\
\text { PSL }\end{array}$ & Plantar test & $\uparrow$ Latency & $\alpha 2-A R$ & Idazoxan (3 $\mu$ g, i.t.) & Mice & Takeuchi et al., 2007 \\
\hline Pregabalin & 30 i.c.v. & & von Frey test & $\uparrow$ Latency & $\alpha 2-A R$ & & & \\
\hline \multirow[t]{2}{*}{ Gabapentin } & 100 mg/kg i.p. & $\begin{array}{l}\text { Neuropathic: } \\
\text { PSL }\end{array}$ & Plantar test & $\uparrow$ Latency & $\alpha 2-A R$ & Yohimbine (1 mg/kg i.p.; 3 g i.t) & Mice & Tanabe et al., 2008 \\
\hline & $100 \mu \mathrm{g}$ i.t. & & von Frey test & $\uparrow$ Latency & & & & \\
\hline \multicolumn{9}{|l|}{ Opioids } \\
\hline \multirow[t]{4}{*}{ Beta-endorphin } & $1 \mathrm{nmol} / 10 \mu$ li.t. & $\begin{array}{l}\text { Heat } \\
\text { stimulation }\end{array}$ & Tail-flick test & $\uparrow$ Latency & $5-\mathrm{HT} 1$ & Spiroxatrine $(15 \mu \mathrm{g} / 10 \mu \mathrm{l})$ & Rat & Crisp et al., 1989 \\
\hline & & & & & $5-\mathrm{HT} 3$ & ICS 205-930 (15 $\mu \mathrm{g} / 10 \mu \mathrm{l})$ & & \\
\hline & & & & & $5-\mathrm{HT} 2$ & Ritanserin $(15 \mu \mathrm{g} / 10 \mu \mathrm{l})$ & & \\
\hline & & & & & $\alpha 2-A R$ & Yohimbine $(15 \mu \mathrm{g} / 10 \mu \mathrm{l})$ & & \\
\hline DAMGO & $1 \mu \mathrm{g} / \mathrm{paw}$ & $\begin{array}{l}\text { PGE2 } \\
\text { (intraplantar) }\end{array}$ & Randall-Selitto & $\begin{array}{l}\uparrow \text { Nociceptive } \\
\text { threshold }\end{array}$ & $\alpha 2-A R$ & Yohimbine $(5,10,20 \mathrm{mg} / \mathrm{paw})$ & Rat & Romero et al., 2012 \\
\hline SCN 80 & $20 \mu \mathrm{g} / \mathrm{paw}$ & & & & $\alpha 2 A-A R$ & 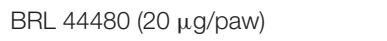 & & \\
\hline \multirow[t]{5}{*}{ Bremazocine } & $20 \mu \mathrm{g} / \mathrm{paw}$ & & & & $\alpha 2 B-A R$ & 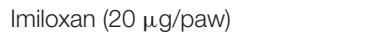 & & \\
\hline & & & & & $\alpha 2 C-A R$ & $\operatorname{RAU}(10,15$, and $20 \mu \mathrm{g} / \mathrm{paw})$ & & \\
\hline & & & & & $\alpha 2 D-A R$ & RX $821002(20 \mu \mathrm{g} / \mathrm{paw})$ & & \\
\hline & & & & & $\alpha 1-A R$ & Prazosin $(0.5,1,2 \mu \mathrm{g} /$ paw $)$ & & \\
\hline & & & & & $\beta-A R$ & Propanolol (150, 300, 600 ng/paw) & & \\
\hline \multirow[t]{2}{*}{ Morphine } & $7.5 \mathrm{nmol} / 10 \mu \mathrm{l} \mathrm{i.t}$ & $\begin{array}{l}\text { Heat } \\
\text { stimulation }\end{array}$ & Tail-flick test & $\uparrow$ Latency & $5-\mathrm{HT} 2$ & Ketanserin (50 nmol, i.t.) & Rat & Kellstein et al., 1988 \\
\hline & & & & & & Methysergide (20 nmol i.t.) & & \\
\hline \multirow[t]{2}{*}{ Fluoxetine } & $\begin{array}{l}10 \text { mg/kg i.p. } \\
\text { (7 days) }\end{array}$ & $\begin{array}{l}\text { Abdominal } \\
\text { constriction }\end{array}$ & Acetic acid test & $\downarrow$ Writhes & $5-\mathrm{HT} 1 \mathrm{~A}$ & Naloxone (5 mg/kg i.p.) & Mice & Singh et al., 2001 \\
\hline & & & & & & Naltrexone (5 mg/kg i.p.) & & \\
\hline \multicolumn{9}{|l|}{ NSAID's } \\
\hline Paracetamol & 400 mg/kg i.p. & & Hot plate test & $\uparrow$ Reaction time & $5-\mathrm{HT} 2$ & {$[3 \mathrm{H}]$ Ketanserin binding } & Rat & Pini et al., 1996 \\
\hline ASA+morphine & $\begin{array}{l}50 \text { mg/kg i.p; } \\
3 \text { mg/kg s.c }\end{array}$ & Inflammatory & $\begin{array}{l}\text { Formalin test } \\
\text { (both phases) }\end{array}$ & $\downarrow$ Flinches & & & & \\
\hline Clonidine & 1, $10 \mu \mathrm{g}$ i.t. & $\begin{array}{l}\text { Heat } \\
\text { stimulation }\end{array}$ & $\begin{array}{l}\text { Noxious heat } \\
\text { stimuli }\end{array}$ & $\uparrow$ Latency & $\alpha 2-A R$ & Ketorolac (50 $\mu$ g i.t.) & Rat & Conklin and Eisenach, 2003 \\
\hline
\end{tabular}


TABLE 3 | Continued

\begin{tabular}{|c|c|c|c|c|c|c|c|c|}
\hline Drugs & Effective Dose & Model of pain & $\begin{array}{l}\text { Nociceptive } \\
\text { test }\end{array}$ & Effect & Receptors & Effect antagonized by & Animal & References \\
\hline Dypirone & $20 \mu \mathrm{g} / \mathrm{paw}$ & $\begin{array}{l}\text { PGE2 } \\
\text { (intraplantar) }\end{array}$ & Randall-Selitto & $\uparrow$ Latency & $\alpha 2-A R$ & Yohimbine $(2.5,5,10,20 \mathrm{mg} / \mathrm{paw})$ & Rat & Silva et al., 2015 \\
\hline \multirow[t]{3}{*}{ Diclofenac } & $40 \mu \mathrm{g} / \mathrm{paw}$ & & & & $\alpha 2 C-A R$ & $\operatorname{RAU}(10,15,20 \mu \mathrm{g} / \mathrm{paw})$ & & \\
\hline & & & & & $\alpha 1-A R$ & Prazosin $(0.5,1,2 \mu \mathrm{g} / \mathrm{paw})$ & & \\
\hline & & & & & $\beta-A R$ & Propanolol (0.3, 0.6, $1.2 \mu \mathrm{g} / \mathrm{paw})$ & & \\
\hline
\end{tabular}

Chlorpheniramine

Ranitidine

$15 \mathrm{mg} / \mathrm{kg}$ i.p.
$15 \mathrm{mg} / \mathrm{kg}$ i.p.
$100 \mathrm{mg} / \mathrm{kg}$, i.p.

$50,100 \mathrm{mg} / \mathrm{kg}$,
i.p. (12 days)
$20,20 \mathrm{mg} / \mathrm{kg}$, i.p.
(12 days)

$$
\text { TNT }
$$

Histidine

Neuropathic: PSL

Promethazine

\section{Vincristine-} mediated neuropathy

Ranitidine

1, 5, 10,

E-162

$20 \mathrm{mg} / \mathrm{kg}$, i.p.

Neuropathic:

$\mathrm{CCl}$

TR-7

1 mg/kg, p.o.

(4 days)

S38093

A-960656

1, 3 mg/kg, p.o.

(11 days)

$0.1,0.3$

$1 \mathrm{mg} / \mathrm{kg}$, p.o.

(12 days)

JNJ7777120

$28,70 \mathrm{mg} / \mathrm{kg}$ i.p.

(8 days)

Neuropathic:

von Frey test

$\uparrow$ Latency

$\mathrm{H} 1$

Acetone test

$\uparrow$ Latency

von Frey test

$\uparrow$ Latency

$\mathrm{H} 2$

Plantar test $\quad \uparrow$ Latency

Pinprick test $\quad \uparrow$ Latency

Acetone test $\quad \uparrow$ Latency

Hot plate test $\quad \uparrow$ Latency

von Frey test $\quad \uparrow$ Latency

\section{Cold plate}

$\uparrow$ Latency

Tail-flick test

Neuropathic:

Randall-Selitto

$\uparrow$ Latency

Neuropathic:

SNL

Inflammatory

Randall-Selitto $\uparrow$ Latency

$\mathrm{H} 3$

H3

$10 \mu \mathrm{g}$ i.t.)

Rat

Khalizzadeh et al., 2018

11

Mepyramine (200 ng/rat i.t. or i.c.v)

Rat

Yu et al., 2016

Jaggi et al., 2017

(n)

$\mathrm{H} 4$

H3

H3

Rat

Chaumette et al., 2018

Cowart et al., 2012

ST-1006

30 and $60 \mu \mathrm{g} \quad$ Neuropathic:

SNI

Plantar test $\quad \uparrow$ Latency $\quad \mathrm{H} 4$

4

Plantar test $\quad \uparrow$ Latency $\quad \mathrm{H} 4$

JNJ 10191584 (6 mg/kg p.o.)

Hsieh et al., 2010

Von Frey test $\quad \uparrow$ Latency

i.c.v.

AR, adrenoceptor; ASA, Acetylsalicylic acid; CCI, chronic constriction injury; i.c.v, intracerebroventricular injection; i.p., intraperitoneal injection; i.t. intrathecal injection; p.o, oral administration; PSL, partial ligation of the sciatic nerve; s.c., subcutaneous injection; SNL, spinal nerve ligation; TNT, tibial nerve transection; SNI, spared nerve injury. 
dysfunction of the reward circuit, which should be studied as a target for the management of chronic pain.

\section{Clinical Approach}

Gabapentinoids are mainly effective in the treatment of chronic neuropathic pain, with gabapentin and pregabalin the drugs considered to most effectively relieve neuropathic pain (e.g., diabetic neuropathy and post-herpetic neuralgia). However, in terms of non-neuropathic pain only pregabalin has been seen to be efficacious in the treatment of fibromyalgia (Wiffen et al., 2013). Most clinical trials indicate that antidepressants and gabapentinoids produce comparable analgesia, mainly in neuropathic pain patients, yet more recent trials attempted to see if analgesia is improved by combining these two drugs. For neuropathic pain (diabetic neuropathy or post-herpetic neuralgia), the combined action of gabapentin and the TCA nortriptyline proved to be more efficacious than either drug alone (Gilron et al., 2009). The combination of pregabalin and duloxetine was well tolerated in diabetic neuropathy patients, although it did not produce better analgesia than either drug alone (Tesfaye et al., 2013). Interestingly, this combination produced a notably better clinical outcome in fibromyalgia when compared to monotherapy (Gilron et al., 2016). There is little data regarding the use of gabapentinoids in pain with a pure inflammatory component, although a recent study indicated that the combination of pregabalin with a NSAID in knee osteoarthritis patients with neuropathic pain was more effective than monotherapy with the NSAID alone (Filatova et al., 2017).

\section{The Combination of Monoamines and Opioids \\ Preclinical Studies}

Opioids are first line treatments for many types of chronic pain and several studies have reported combined effects between the opioid and monoaminergic systems (Table 3; Schroder et al., 2011; Bravo et al., 2017). Opioid receptor agonists induce NA release at supraspinal, spinal and peripheral sites (Wigdor and Wilcox, 1987; Grabow et al., 1999) where they would exert analgesic actions. Early studies reported that $\alpha 2$-ARs mediate analgesia of $\beta$-endorphin at the spinal cord (Crisp et al., 1989 ) and that naloxone antagonizes intrathecal NA-induced anti-nociception, suggesting that opioids are involved in NA spinal analgesia. Interestingly, co-administration of TCAs and morphine enhance analgesia in mice (Kellstein et al., 1984; Hwang and Wilcox, 1987; Gray et al., 1999; Reimann et al., 1999), and $\alpha 2$-ARs are thought to be responsible for this synergistic action (Stone et al., 1999). Recent studies demonstrated that opioids and cannabinoids induce peripheral anti-nociception by releasing NA, which activates the $\alpha 2-, \alpha 1-$ and $\beta$-ARs (Romero et al., 2012).

However, there are is some controversy as to whether opioids mediate spinal anti-nociception via NA or 5-HT. Inhibition of both NA and 5-HT reuptake appears to induce analgesic synergy with morphine in the formalin test, although excess 5HT may stimulate 5-HT3 receptors and reduces this synergy (Shen et al., 2013). Alternatively, there is evidence that opioids mediate spinal 5-HT anti-nociception (Kellstein et al., 1988;
Kishimoto et al., 2001) and moreover, the anti-nociceptive effect of fluoxetine in the acetic acid test is sensitive to blockade by naloxone (Singh et al., 2001), suggesting a clear opioid component in this effect.

\section{Clinical Approach}

Combining monoamines and opioids enhances analgesia in animal models, although in only one clinical trial were benefits reported using the combination of nortriptyline and morphine (Gilron et al., 2015). Unfortunately, this combination induces the classic adverse effects of opioids (constipation, dry mouth and somnolence) and could compromise patient safety. Hence, drugs are being designed that offer a reasonable safety profile and that combine monoaminergic and opioid actions. This is the case of tramadol, a mu-opioid agonist and SNRI, and tapentadol, a potent mu-opioid agonist and NA reuptake inhibitor. These drugs relieve certain types of chronic pain but they do not appear to have a reliable effect on neuropathies (Silverfield et al., 2002; Duehmke et al., 2017), which has led to them being more commonly recommend as second-line therapy.

\section{NSAIDs \\ Preclinical Studies}

Preclinical and clinical studies demonstrated the efficacy of NSAIDs, mainly in chronic inflammatory pain (Cohen and Harris, 1987; Vo et al., 2009), and they are commonly prescribed for acute and persistent inflammatory pain. The peripheral inhibition of prostaglandins is not the only analgesic mechanism of action, given its central action on the noradrenergic and serotonergic system (Table 3). Acetyl salicylic acid (ASA) induced analgesia is accompanied by an increase in the turnover of 5HT, NA and DA (Bensemana and Gascon, 1978), and a potent relationship has been described between 5-HT and NSAIDs. Acute administration of phenazone decreases 5-HT binding, whereas chronic treatment provokes an increase in 5-HT binding sites in the pontine and cortical areas, induced by an increase in 5-HT (Sandrini et al., 1998). The anti-nociceptive effect of ASA was impeded by i.p. pre-treatment with a 5-HT neurotoxin in the hot plate test (Pini et al., 1996). Moreover, ASA potentiated the anti-nociceptive effect of morphine in the second phase of the formalin test, accompanied by an increase in extracellular 5-HT and a decrease in 5-HT2 receptors in the cortex (Sandrini et al., 1998). Similar findings were reported with the combination of paracetamol and morphine. More recently, adrenergic receptors have been implicated in the anti-nociceptive effect of NSAIDs. Thus, intrathecal ketorolac enhances the effect of clonidine against a noxious heat stimulus in rats, yet it lacks efficacy when administered alone (Conklin and Eisenach, 2003). Additionally, the peripheral depletion of NA prevented the anti-nociceptive effect of dipyrone and diclofenac in a model of inflammatory pain, while prazosin (an $\alpha 1$ antagonist) and propranolol (a $\beta$-adrenergic antagonist) blocked the anti-nociceptive effect of dipyrone and diclofenac (Silva et al., 2015), suggesting that NSAID analgesia is mediated by adrenergic receptors. There are no relevant studies into the analgesic efficacy of NSAIDs in animal models of neuropathic pain (Lashbrook et al., 1999), yet further preclinical studies into both chronic neuropathic 
and inflammatory pain should elucidate the possible interaction between NSAIDs and monoamines.

\section{Clinical Approach}

NSAIDs are routinely prescribed for the management of chronic inflammatory pain since their main mechanism of action involves the peripheral inhibition of inflammatory mediators (prostaglandins), provoking weaker peripheral sensitization. However, the oral NSAIDs used have more severe adverse effects when administered chronically (peptic ulcer disease, acute renal failure and stroke/myocardial infarction) and thus, topical rather than oral administration is recommended. In clinical practice, NSAIDs have relevant peripheral activity but recently, there is recent evidence that they may facilitate activation of the descending inhibitory circuitry at the level of the spinal cord and brain (Hodkinson et al., 2015). In terms of neuropathic pain, NSAIDs have no effect and there is only modest evidence of efficacy in patients with chronic low back pain (Enthoven et al., 2017).

\section{Histaminergic Drugs}

Recent, preclinical findings support the use of ligands of histamine receptors as analgesics in neuropathic and inflammatory pain (Table 3; Obara et al., 2019). For example, the systemic administration of $\mathrm{H} 1$ and $\mathrm{H} 2$ receptor antagonists (like chlorpheniramine and ranitidine, respectively) produces anti-allodynic effects in a model of neuropathic pain in rats (Khalilzadeh et al., 2018). Interestingly, several studies suggest that $\mathrm{H} 1$ but not $\mathrm{H} 2$ receptors are involved in the development of hypersensitivity following partial ligation of the sciatic nerve and in vincristine-induced models of neuropathic pain (Yu et al., 2016; Jaggi et al., 2017).

Although most studies suggest that the systemic use of histamine $\mathrm{H} 3$ receptor antagonists produces inhibitory effects on nociceptive responses in neuropathic pain (Farzin and Nosrati, 2007; Chaumette et al., 2018; Popiolek-Barczyk et al., 2018), there is evidence that activation of $\mathrm{H} 3$ receptors has inhibitory effects on pain (Cannon et al., 2003). Interestingly, chronic oral administration of S38093 or A-960656, selective H3 receptor antagonist/inverse agonists, increased the paw withdrawal threshold to mechanical stimuli in neuropathic and inflammatory pain models, showing similar effects to gabapentin and/or pregabalin (Cowart et al., 2012; Chaumette et al., 2018). Moreover, the analgesic effect of S38093 is thought to be partially mediated by a2-AR desensitization in the LC, suggesting that the noradrenergic system is crucial for $\mathrm{H} 3$ antagonists to produce antinociception (Chaumette et al., 2018). In fact, bilateral lesions of the LC and spinal cord transection completely inhibited the effects of $\mathrm{H} 3$ antagonist, on spontaneous and evoked firing of spinal neurons in neuropathic rats (McGaraughty et al., 2012). Like H3 receptors, H4 receptor agonists and antagonists have different effects on the nociceptive response. Thus, systemic administration of JNJ 7777120, a histamine $\mathrm{H} 4$ receptor antagonist, reduced mechanical hypersensitivity in chronic constriction injury (CCI) and SNL neuropathic models (Hsieh et al., 2010). In addition, single doses of the selective H4 receptor antagonist TR-7 elicit a strong analgesic effect (PopiolekBarczyk et al., 2018). By contrast, local ICV administration of the H4 receptor agonists ST-1006 and VUF8430, reduced nociceptive thresholds in mice with neuropathic pain (Sanna et al., 2015).

Overall, there appears to be a clear effect of histamine in chronic pain that depends on multiple factors, such as the localization of the receptors, or the affinity and selectivity of ligands for histamine receptors (Obara et al., 2019). Thus, a better understanding of the histaminergic system in pain modulation will help us to identify histaminergic targets that could lead to more efficient pharmacological therapy for neuropathic pain.

\section{DISCUSSION}

The monoaminergic system is important in both the healthy state and in pain modulation, the correct balance between the excitatory and inhibitory inputs on descending pain pathways producing analgesia. However, when biological pain shifts to pathological pain, the monoaminergic system suffers alterations that would contribute to the sensitization and maintenance of pain. To search for pharmacological new targets, the monoaminergic mechanisms underlying chronic pain are of great interest. In fact, chronic pain, and explicitly neuropathic pain, is treated with medications that affect monoamines. Antidepressants are drugs that effect monoamines directly, although they were designed to treat depression and they are currently used as first line treatments for neuropathic pain due to their intrinsic analgesic action. In recent decades, antidepressants have been seen to have greater clinical efficacy in neuropathic pain than in inflammatory pain (Finnerup et al., 2005; Sindrup et al., 2005). From an etiological point of view, neuropathic pain is due to lesion or dysfunction in the PNS/CNS that causes monoaminergic dysfunction, yet inflammatory pain is caused by the action of inflammatory mediators on nociceptors in the periphery and subsequently, changes in the excitability of central neurons. The analgesic mechanism of action of antidepressants is thought to involve inhibition of both NA and 5-HT reuptake at spinal and brain levels, and the subsequent modulation of descending pain pathways. Thus, it is plausible that antidepressants exert positive clinical outcomes in pain with a neuropathic component.

In addition, antidepressants exhibit significant analgesia in chronic pain, yet moderate efficacy against acute pain (Poree et al., 1998; Sawynok et al., 2001; Paqueron et al., 2003; Mico et al., 2006). As indicated, the increase of $\alpha 2$ AR efficacy at the spinal level seems to promote better analgesia of antidepressants (Stone et al., 1999; Bantel et al., 2005). Moreover, supraspinal noradrenergic centers like the LC are altered in chronic pain, alterations that are relevant for the development of chronic pain-related co-morbidities like anxiety and depression (Alba-Delgado et al., 2013, 2018), and which are commonly treated with antidepressants. Although antidepressants mainly act through the NA and 5-HT system, the repercussion of each system on anti-nociception remains unclear. Antidepressants that affect both noradrenaline and 
serotonin levels, like duloxetine and amitriptyline, are more potent and efficient than SSRIs in relieving chronic pain (Marchand et al., 2003; Finnerup et al., 2005). Considering data from animal studies, milnacipran (a SNRI) but not paroxetine (a SSRI) attenuates mechanical hypersensitivity in a model of neuropathic pain (SNL: Bomholt et al., 2005). Furthermore, the anti-nociceptive effect of drugs that act selectively on 5HT (e.g., SSRIs) is less potent in chronic than in acute pain (Bardin et al., 2000; Pichon et al., 2010). This phenomenon could be explained by the pro-nociceptive effect of spinal 5-HT receptors in some states of chronic pain and by the alterations to 5-HT2A receptors observed in neuropathic pain (Honda et al., 2006; Pichon et al., 2010). These studies reveal the importance of studying 5-HT receptor agonists/antagonists in pain management. Currently, no drugs used to manage chronic pain target 5-HT receptors, except the 5-HT1B/1D agonists used to treat chronic migraine and headache. Interestingly, drugs that mainly inhibit noradrenaline reuptake, like DMI and maprotiline, are not a good choice for pain relief, as reflected by their moderate relief in the treatment of human neuropathic pain (Max et al., 1991, 1992; Vrethem et al., 1997), revealing the importance of increasing both extracellular NA and 5-HT.

Clinical evidence suggests TCAs are more efficacious in several chronic pain conditions. In particular, amytriptiline is considered the gold standard to manage neuropathic pain irrespective of its etiology (Saarto and Wiffen, 2007), although the main drawback of these drugs are their adverse effects. However, optimum analgesia is usually achieved at lower doses than those required for their antidepressant activity and thus, such side effects occur less frequently. Non-tricyclic antidepressants are thought to be safer than TCAs, and duloxetine is the first antidepressant approved by the FDA to treat neuropathic pain, considered the best election for peripheral diabetic neuropathic pain (Kaur et al., 2011; King et al., 2015). Less convincing results have been obtained in terms of the selective action on 5-HT receptors, yet preclinical studies indicate that the coadministration of antidepressants with certain 5-HT receptor antagonists (e.g., those of the 5-HT1A receptor) potentiates the analgesic effect of these drugs (Ardid et al., 2001; Singh et al., 2001; Anjaneyulu and Chopra, 2004). Recently, other atypical antidepressants like melatonin and agomelatonine have been shown to have promising analgesic effects to treat neuropathic pain, acting via MT1/MT2 melatonin receptors and the monoaminergic system (Ambriz-Tututi et al., 2009; Lopez-Canul et al., 2015; Chenaf et al., 2017). Moreover, the introduction of TRIs represents a new strategy that can be explored in the management of chronic pain. As indicated, chronic pain induces important changes in the NA, 5-HT and DA system, and as such, these drugs would maintain optimal levels of these neurotransmitters that allow a longer interaction with the appropriate receptors.

In addition to antidepressants, other drugs that directly and indirectly effect the monoaminergic system are also used to treat chronic pain. Thus, atypical opioids that combine monoaminergic and opioid effects are more efficacious than other opioids in neuropathic pain models (Christoph et al., 2007;
Meske et al., 2014). In fact, tramadol tapentadol are situated on the second third step of the WHO analgesic scale (WHO, Bravo et al., 2017). Conversely, gabapentinoids mediate analgesia via spinal $\alpha 2$-ARs (Takasu et al., 2006) and they have been approved by the FDA to treat neuropathic pain (Goodman and Brett, 2019). The efficacy of gabapentinoids is comparable to that of antidepressant drugs in clinical trials, yet they differ in safety and tolerability (Morello et al., 1999; Sindrup and Jensen, 2000). They now represent an alternative for neuropathic pain patients for whom amytriptiline is contraindicated. Finally, NSAIDs are drugs that act on the monoaminergic system, yet with limited information regarding their mechanism of action. In general, NSAIDs seem to potentiate the analgesic effect of drugs like morphine and tramadol, and recently, their activity was associated with $\alpha 1$ - and $\beta$-ARs (Silva et al., 2015). Clinically, there is a little evidence of the potency and efficacy of NSAIDs in chronic pain, or of their use in combination with other drugs (Ong et al., 2007). Finally, although there are no histaminergic drugs that relieve chronic pain, preclinical studies indicate that it is time to reconsider the histamine system as a therapeutic target for the management of inflammatory and neuropathic pain.

In conclusion, an extracellular increase in NA and 5-HT significantly helps relieve chronic pain. Indeed, chronic neuropathic pain is commonly treated with TCA and SNRI antidepressants, and by gabapentinoid drugs when antidepressants are contraindicated. Given that the monoaminergic system is closely linked to disorders like depression and anxiety, clinical situations frequently associated with chronic pain, the monoaminergic system is a pharmacological target that could help treat the sensory and emotional aspects of chronic pain. However, despite the promising preclinical and clinical results obtained, complete relief from chronic pain via the monoaminergic system remains a challenge. Thus, there is a need for further preclinical and clinical studies to further assess the selective targeting of the monoaminergic system to achieve successful analgesia for the treatment of chronic pain.

\section{AUTHOR CONTRIBUTIONS}

JM and LB performed the design of the structure of manuscript, analyzed the literature searching, wrote and revised several drafts of the manuscript. Also, they revised tables and contribute to perform the Figure 1. ML-T wrote the first part of the manuscript, revised the first draft of manuscript and elaborated the tables and the figure. EB analyzed the literature searching and revised the tables and the final draft of the manuscript.

\section{FUNDING}

This study was supported by grants co-financed by the "Fondo Europeo de Desarrollo Regional" (FEDER)-UE “A way to build Europe" from the "Ministerio de Economía y Competitividad" 
(Ministerio de Ciencia, Innovación y Universidades:Salud RTI2018-099778-B-I00) and the "Ministerio de Salud-Instituto de Carlos III (PI18/01691), as well as funding from the "Consejería de Salud de la Junta de Andalucía" (PI-01342018); the "Programa Operativo de Andalucía FEDER, Iniciativa Territorial Integrada ITI 2014-2020 Consejería Salud, Junta de Andalucía" (PI-0080-2017); the "Consejería de Economía, Innovación, Ciencia y Empleo de la Junta de Andalucía” (CTS-510); and the "Centro de Investigación Biomédica en

\section{REFERENCES}

Aimone, L. D., and Gebhart, G. F. (1986). Stimulation-produced spinal inhibition from the midbrain in the rat is mediated by an excitatory amino acid neurotransmitter in the medial medulla. J. Neurosci. 6, 1803-1813. doi: 10. 1523/jneurosci.06-06-01803.1986

Alba-Delgado, C., Borges, G., Sanchez-Blazquez, P., Ortega, J. E., Horrillo, I., Mico, J. A., et al. (2012). The function of alpha-2-adrenoceptors in the rat locus coeruleus is preserved in the chronic constriction injury model of neuropathic pain. Psychopharmacology 221, 53-65. doi: 10.1007/s00213-011-2542-7

Alba-Delgado, C., Llorca-Torralba, M., Horrillo, I., Ortega, J. E., Mico, J. A., Sanchez-Blazquez, P., et al. (2013). Chronic pain leads to concomitant noradrenergic impairment and mood disorders. Biol. Psychiatry 73, 54-62. doi: 10.1016/j.biopsych.2012.06.033

Alba-Delgado, C., Llorca-Torralba, M., Mico, J. A., and Berrocoso, E. (2018). The onset of treatment with the antidepressant desipramine is critical for the emotional consequences of neuropathic pain. Pain 159, 2606-2619. doi: 10. 1097/j.pain.0000000000001372

Aley, K. O., Martin, A., McMahon, T., Mok, J., Levine, J. D., and Messing, R. O. (2001). Nociceptor sensitization by extracellular signal-regulated kinases. J. Neurosci. 21, 6933-6939. doi: 10.1523/jneurosci.21-17-06933.2001

Alhaider, A. A., and Wilcox, G. L. (1993). Differential roles of 5hydroxytryptamine1A and 5-hydroxytryptamine1B receptor subtypes in modulating spinal nociceptive transmission in mice. J. Pharmacol. Exp. Ther. 265, 378-385.

Ali, H., Choi, O. H., Fraundorfer, P. F., Yamada, K., Gonzaga, H. M., and Beaven, M. A. (1996). Sustained activation of phospholipase D via adenosine A3 receptors is associated with enhancement of antigen- and $\mathrm{Ca}(2+)$-ionophoreinduced secretion in a rat mast cell line. J. Pharmacol. Exp. Ther. 276, 837-845.

Amaya-Castellanos, E., Pineda-Farias, J. B., Castaneda-Corral, G., Vidal-Cantu, G. C., Murbartian, J., Rocha-Gonzalez, H. I., et al. (2011). Blockade of 5-HT7 receptors reduces tactile allodynia in the rat. Pharmacol. Biochem. Behav. 99, 591-597. doi: 10.1016/j.pbb.2011.06.005

Ambriz-Tututi, M., Rocha-Gonzalez, H. I., Cruz, S. L., and Granados-Soto, V. (2009). Melatonin: a hormone that modulates pain. Life Sci. 84, 489-498. doi: 10.1016/j.lfs.2009.01.024

Anderberg, U. M., Marteinsdottir, I., and Von Knorring, L. (2000). Citalopram in patients with fibromyalgia-a randomized, double-blind, placebo-controlled study. Eur. J. Pain 4, 27-35. doi: 10.1053/eujp.1999.0148

Anjaneyulu, M., and Chopra, K. (2004). Fluoxetine attenuates thermal hyperalgesia through 5-HT1/2 receptors in streptozotocin-induced diabetic mice. Eur. J. Pharmacol. 497, 285-292. doi: 10.1016/j.ejphar.2004.06.063

Ansah, O. B., Leite-Almeida, H., Wei, H., and Pertovaara, A. (2007). Striatal dopamine D2 receptors attenuate neuropathic hypersensitivity in the rat. Exp. Neurol. 205, 536-546. doi: 10.1016/j.expneurol.2007.03.010

Ardid, D., Alloui, A., Brousse, G., Jourdan, D., Picard, P., Dubray, C., et al. (2001). Potentiation of the antinociceptive effect of clomipramine by a $5-\mathrm{ht}(1 \mathrm{~A})$ antagonist in neuropathic pain in rats. Br. J. Pharmacol. 132, 1118-1126. doi: 10.1038/sj.bjp.0703897

Asano, T., Dohi, S., Ohta, S., Shimonaka, H., and Iida, H. (2000). Antinociception by epidural and systemic alpha(2)-adrenoceptor agonists and their binding affinity in rat spinal cord and brain. Anesth. Analg. 90, 400-407. doi: 10.1097/ 00000539-200002000-00030

Baik, J. H. (2013). Dopamine signaling in reward-related behaviors. Front. Neural Circ. 7:152. doi: 10.3389/fncir.2013.00152
Red de Salud Mental - CIBERSAM" (CB/07/09/0033) and a Young Investigator Grant from the Brain Behavior Research Foundation (NARSAD23982).

\section{ACKNOWLEDGMENTS}

The authors thank Mark Sefton of BIOMEDRED SL. Madrid, Spain for correcting the English language in this article.

Bannister, K., Bee, L. A., and Dickenson, A. H. (2009). Preclinical and early clinical investigations related to monoaminergic pain modulation. Neurotherapeutics 6 , 703-712. doi: 10.1016/j.nurt.2009.07.009

Bannister, K., and Dickenson, A. H. (2016). What do monoamines do in pain modulation? Curr. Opin. Support. Palliat. Care 10, 143-148. doi: 10.1097/SPC. 0000000000000207

Bannister, K., and Dickenson, A. H. (2017). The plasticity of descending controls in pain: translational probing. J. Physiol. 595, 4159-4166. doi: 10.1113/JP274165

Bannister, K., Lockwood, S., Goncalves, L., Patel, R., and Dickenson, A. H. (2017). An investigation into the inhibitory function of serotonin in diffuse noxious inhibitory controls in the neuropathic rat. Eur. J. Pain 21, 750-760. doi: 10.1002/ ejp.979

Bantel, C., Eisenach, J. C., Duflo, F., Tobin, J. R., and Childers, S. R. (2005). Spinal nerve ligation increases alpha2-adrenergic receptor G-protein coupling in the spinal cord. Brain Res. 1038, 76-82. doi: 10.1016/j.brainres.2005.01.016

Bardin, L., Jourdan, D., Alloui, A., Lavarenne, J., and Eschalier, A. (1997). Differential influence of two serotonin 5-HT3 receptor antagonists on spinal serotonin-induced analgesia in rats. Brain Res. 765, 267-272. doi: 10.1016/ s0006-8993(97)00566-0

Bardin, L., Schmidt, J., Alloui, A., and Eschalier, A. (2000). Effect of intrathecal administration of serotonin in chronic pain models in rats. Eur. J. Pharmacol. 409, 37-43. doi: 10.1016/s0014-2999(00)00796-2

Bardin, L., Tarayre, J. P., Koek, W., and Colpaert, F. C. (2001). In the formalin model of tonic nociceptive pain, 8-OH-DPAT produces 5-HT1A receptormediated, behaviorally specific analgesia. Eur. J. Pharmacol. 421, 109-114. doi: 10.1016/s0014-2999(01)01029-9

Baron, R., Schwarz, K., Kleinert, A., Schattschneider, J., and Wasner, G. (2001). Histamine-induced itch converts into pain in neuropathic hyperalgesia. Neuroreport 12, 3475-3478. doi: 10.1097/00001756-20011116000020

Bee, L. A., and Dickenson, A. H. (2008). Descending facilitation from the brainstem determines behavioural and neuronal hypersensitivity following nerve injury and efficacy of pregabalin. Pain 140, 209-223. doi: 10.1016/j.pain.2008. 08.008

Bensemana, D., and Gascon, A. L. (1978). Relationship between analgesia and turnover of brain biogenic amines. Can. J. Physiol. Pharmacol. 56, 721-730. doi: 10.1139/y78-115

Bernard, J. M., Kick, O., and Bonnet, F. (1994). Which way for the administration of alpha 2-adrenergic agents to obtain the best analgesia?. Cah. Anesthesiol. 42, 223-228.

Berrocoso, E., and Mico, J. A. (2007). In vivo effect of venlafaxine on locus coeruleus neurons: role of opioid, alpha(2)-adrenergic, and 5hydroxytryptamine(1A) receptors. J. Pharmacol. Exp. Ther. 322, 101-107. doi: 10.1124/jpet.107.120915

Bohren, Y., Tessier, L. H., Megat, S., Petitjean, H., Hugel, S., Daniel, D., et al. (2013). Antidepressants suppress neuropathic pain by a peripheral beta2-adrenoceptor mediated anti-TNFalpha mechanism. Neurobiol. Dis. 60, 39-50. doi: 10.1016/j. nbd.2013.08.012

Bomholt, S. F., Mikkelsen, J. D., and Blackburn-Munro, G. (2005). Antinociceptive effects of the antidepressants amitriptyline, duloxetine, mirtazapine and citalopram in animal models of acute, persistent and neuropathic pain. Neuropharmacology 48, 252-263. doi: 10.1016/j.neuropharm.2004.09.012

Bossut, D. F., and Perl, E. R. (1995). Effects of nerve injury on sympathetic excitation of A delta mechanical nociceptors. J. Neurophysiol. 73, 1721-1723. doi: 10.1152/jn.1995.73.4.1721 
Brandt, S. A., and Livingston, A. (1990). Receptor changes in the spinal cord of sheep associated with exposure to chronic pain. Pain 42, 323-329. doi: 10.1016/0304-3959(90)91145-9

Bravo, L., Mico, J. A., and Berrocoso, E. (2017). Discovery and development of tramadol for the treatment of pain. Expert Opin. Drug Discov. 12, 1281-1291. doi: 10.1080/17460441.2017.1377697

Brown, R. E., Stevens, D. R., and Haas, H. L. (2001). The physiology of brain histamine. Prog. Neurobiol. 63, 637-672. doi: 10.1016/s0301-0082(00)00039-3

Brown, S., Johnston, B., Amaria, K., Watkins, J., Campbell, F., Pehora, C., et al. (2016). A randomized controlled trial of amitriptyline versus gabapentin for complex regional pain syndrome type I and neuropathic pain in children. Scand. J. Pain 13, 156-163. doi: 10.1016/j.sjpain.2016.05.039

Bruinstroop, E., Cano, G., Vanderhorst, V. G., Cavalcante, J. C., Wirth, J., SenaEsteves, M., et al. (2012). Spinal projections of the A5, A6 (locus coeruleus), and A7 noradrenergic cell groups in rats. J. Comp. Neurol. 520, 1985-2001. doi: $10.1002 / \mathrm{cne} .23024$

Buerkle, H., and Yaksh, T. L. (1998). Pharmacological evidence for different alpha 2 -adrenergic receptor sites mediating analgesia and sedation in the rat. $\mathrm{Br}$. J. Anaesth. 81, 208-215. doi: 10.1093/bja/81.2.208

Burch, R. (2019). Antidepressants for preventive treatment of migraine. Curr. Treat. Options Neurol. 21:18. doi: 10.1007/s11940-019-0557-2

Burkey, A. R., Carstens, E., and Jasmin, L. (1999). Dopamine reuptake inhibition in the rostral agranular insular cortex produces antinociception. J. Neurosci. 19, 4169-4179. doi: 10.1523/jneurosci.19-10-04169.1999

Calvo, M., and Bennett, D. L. (2012). The mechanisms of microgliosis and pain following peripheral nerve injury. Exp. Neurol. 234, 271-282. doi: 10.1016/j. expneurol.2011.08.018

Cannon, K. E., Nalwalk, J. W., Stadel, R., Ge, P., Lawson, D., Silos-Santiago, I., et al. (2003). Activation of spinal histamine $\mathrm{H} 3$ receptors inhibits mechanical nociception. Eur. J. Pharmacol. 470, 139-147. doi: 10.1016/s0014-2999(03) 01737-0

Carboni, E., and Silvagni, A. (2004). Dopamine reuptake by norepinephrine neurons: exception or rule? Crit. Rev. Neurobiol. 16, 121-128. doi: 10.1615/ critrevneurobiol.v16.i12.130

Cardenas, D. D., Warms, C. A., Turner, J. A., Marshall, H., Brooke, M. M., and Loeser, J. D. (2002). Efficacy of amitriptyline for relief of pain in spinal cord injury: results of a randomized controlled trial. Pain 96, 365-373. doi: 10.1016/ s0304-3959(01)00483-3

Cardoso-Cruz, H., Dourado, M., Monteiro, C., Matos, M. R., and Galhardo, V. (2014). Activation of dopaminergic D2/D3 receptors modulates dorsoventral connectivity in the hippocampus and reverses the impairment of working memory after nerve injury. J. Neurosci. 34, 5861-5873. doi: 10.1523/ JNEUROSCI.0021-14.2014

Cervantes-Durán, C., Rocha-Gonzalez, H. I., and Granados-Soto, V. (2013). Peripheral and spinal 5-HT receptors participate in the pronociceptive and antinociceptive effects of fluoxetine in rats. Neuroscience 252, 396-409. doi: 10.1016/j.neuroscience.2013.08.022

Chang, P. C., Pollema-Mays, S. L., Centeno, M. V., Procissi, D., Contini, M., Baria, A. T., et al. (2014). Role of nucleus accumbens in neuropathic pain: linked multi-scale evidence in the rat transitioning to neuropathic pain. Pain 155, 1128-1139. doi: 10.1016/j.pain.2014.02.019

Chaumette, T., Chapuy, E., Berrocoso, E., Llorca-Torralba, M., Bravo, L., Mico, J. A., et al. (2018). Effects of S 38093, an antagonist/inverse agonist of histamine $\mathrm{H} 3$ receptors, in models of neuropathic pain in rats. Eur. J. Pain 22, 127-141. doi: 10.1002/ejp.1097

Chen, M., Hoshino, H., Saito, S., Yang, Y., and Obata, H. (2017). Spinal dopaminergic involvement in the antihyperalgesic effect of antidepressants in a rat model of neuropathic pain. Neurosci. Lett. 649, 116-123. doi: 10.1016/j. neulet.2017.04.017

Chenaf, C., Chapuy, E., Libert, F., Marchand, F., Courteix, C., Bertrand, M., et al. (2017). Agomelatine: a new opportunity to reduce neuropathic pain-preclinical evidence. Pain 158, 149-160. doi: 10.1097/j.pain.0000000000000738

Choucair-Jaafar, N., Beetz, N., Gilsbach, R., Yalcin, I., Waltisperger, E., FreundMercier, M. J., et al. (2011). Cardiovascular effects of chronic treatment with a beta2-adrenoceptor agonist relieving neuropathic pain in mice. Neuropharmacology 61, 51-60. doi: 10.1016/j.neuropharm.2011.02.015

Choucair-Jaafar, N., Yalcin, I., Rodeau, J. L., Waltisperger, E., Freund-Mercier, M. J., and Barrot, M. (2009). Beta2-adrenoceptor agonists alleviate neuropathic allodynia in mice after chronic treatment. Br. J. Pharmacol. 158, 1683-1694. doi: 10.1111/j.1476-5381.2009.00510.x

Christoph, T., Kogel, B., Strassburger, W., and Schug, S. A. (2007). Tramadol has a better potency ratio relative to morphine in neuropathic than in nociceptive pain models. Drugs R D 8, 51-57. doi: 10.2165/00126839-200708010-00005

Coffeen, U., Lopez-Avila, A., Ortega-Legaspi, J. M., Del Angel, R., Lopez-Munoz, F. J., and Pellicer, F. (2008). Dopamine receptors in the anterior insular cortex modulate long-term nociception in the rat. Eur. J. Pain 12, 535-543. doi: 10.1016/j.ejpain.2007.08.008

Cohen, K. L., and Harris, S. (1987). Efficacy and safety of nonsteroidal antiinflammatory drugs in the therapy of diabetic neuropathy. Arch. Intern. Med. 147, 1442-1444. doi: 10.1001/archinte.147.8.1442

Cok, O. Y., Eker, H. E., Yalcin, I., Barrot, M., and Aribogan, A. (2010). Is there a place for beta-mimetics in clinical management of neuropathic pain? Salbutamol therapy in six cases. Anesthesiology 112, 1276-1279. doi: 10.1097/ aln.0b013e3181d 40399

Colpaert, F. C. (2006). 5-HT(1A) receptor activation: new molecular and neuroadaptive mechanisms of pain relief. Curr. Opin. Investig. Drugs 7, 40-47.

Colpaert, F. C., Deseure, K., Stinus, L., and Adriaensen, H. (2006). High-efficacy 5-hydroxytryptamine 1A receptor activation counteracts opioid hyperallodynia and affective conditioning. J. Pharmacol. Exp. Ther. 316, 892-899. doi: 10.1124/ jpet.105.095109

Coluzzi, F., Fornasari, D., Pergolizzi, J., and Romualdi, P. (2017). From acute to chronic pain: tapentadol in the progressive stages of this disease entity. Eur. Rev. Med. Pharmacol. Sci. 21, 1672-1683.

Conklin, D. R., and Eisenach, J. C. (2003). Intrathecal ketorolac enhances antinociception from clonidine. Anesth. Analg. 96, 191-194. doi: 10.1097/ 00000539-200301000-00040

Connelly, W. M., Shenton, F. C., Lethbridge, N., Leurs, R., Waldvogel, H. J., Faull, R. L., et al. (2009). The histamine H4 receptor is functionally expressed on neurons in the mammalian CNS. Br. J. Pharmacol. 157, 55-63. doi: 10.1111/ j.1476-5381.2009.00227.x

Cowart, M., Hsieh, G., Black, L. A., Zhan, C., Gomez, E. J., Pai, M., et al. (2012). Pharmacological characterization of A-960656, a histamine $\mathrm{H}(3)$ receptor antagonist with efficacy in animal models of osteoarthritis and neuropathic pain. Eur. J. Pharmacol. 684, 87-94. doi: 10.1016/j.ejphar.2012.03.048

Crisp, T., Stafinsky, J. L., Hess, J. E., and Uram, M. (1989). Spinal beta-endorphin analgesia involves an interaction with local monoaminergic systems. Eur. J. Pharmacol. 160, 211-217. doi: 10.1016/0014-2999(89)90493-7

Crisp, T., Stafinsky, J. L., Uram, M., Perni, V. C., Weaver, M. F., and Spanos, L. J. (1991). Serotonin contributes to the spinal antinociceptive effects of morphine. Pharmacol. Biochem. Behav. 39, 591-595. doi: 10.1016/0091-3057(91)90133-m

Davis, K. D., Treede, R. D., Raja, S. N., Meyer, R. A., and Campbell, J. N. (1991). Topical application of clonidine relieves hyperalgesia in patients with sympathetically maintained pain. Pain 47, 309-317. doi: 10.1016/03043959(91)90221-i

Dawson, L. F., Phillips, J. K., Finch, P. M., Inglis, J. J., and Drummond, P. D. (2011). Expression of alpha1-adrenoceptors on peripheral nociceptive neurons. Neuroscience 175, 300-314. doi: 10.1016/j.neuroscience.2010.11.064

Dogrul, A., and Uzbay, I. T. (2004). Topical clonidine antinociception. Pain 111, 385-391. doi: 10.1016/j.pain.2004.07.020

Drummond, P. D., Drummond, E. S., Dawson, L. F., Mitchell, V., Finch, P. M., Vaughan, C. W., et al. (2014). Upregulation of $\alpha 1$-adrenoceptors on cutaneous nerve fibres after partial sciatic nerve ligation and in complex regional pain syndrome type II. Pain 155, 606-616. doi: 10.1016/j.pain.2013.12.021

Duehmke, R. M., Derry, S., Wiffen, P. J., Bell, R. F., Aldington, D., and Moore, R. A. (2017). Tramadol for neuropathic pain in adults. Cochrane Database Syst. Rev. 6:CD003726. doi: 10.1002/14651858.CD003726.pub4

Eisenach, J. C., D'angelo, R., Taylor, C., and Hood, D. D. (1994). An isobolographic study of epidural clonidine and fentanyl after cesarean section. Anesth. Analg. 79, 285-290.

Eisenach, J. C., Dupen, S., Dubois, M., Miguel, R., and Allin, D. (1995). Epidural clonidine analgesia for intractable cancer pain. The epidural clonidine study group. Pain 61, 391-399. doi: 10.1016/0304-3959(94)00209-w

Enthoven, W. T. M., Roelofs, P. D., and Koes, B. W. (2017). NSAIDs for chronic low back pain. JAMA 317, 2327-2328. doi: 10.1001/jama.2017.4571

Erfanparast, A., Tamaddonfard, E., Farshid, A. A., and Khalilzadeh, E. (2010). Effect of microinjection of histamine into the dorsal hippocampus on the orofacial 
formalin-induced pain in rats. Eur. J. Pharmacol. 627, 119-123. doi: 10.1016/j. ejphar.2009.10.063

Ernberg, M., Lundeberg, T., and Kopp, S. (2000). Effect of propranolol and granisetron on experimentally induced pain and allodynia/hyperalgesia by intramuscular injection of serotonin into the human masseter muscle. Pain 84, 339-346. doi: 10.1016/s0304-3959(99)00221-3

Farzin, D., and Nosrati, F. (2007). Modification of formalin-induced nociception by different histamine receptor agonists and antagonists. Eur. Neuropsychopharmacol. 17, 122-128 doi: 10.1016/j.euroneuro.2006.03.005

Fields, H. (2004). State-dependent opioid control of pain. Nat. Rev. Neurosci. 5, 565-575. doi: 10.1038/nrn1431

Filatova, E. S., Turovskaya, E. F., and Alekseeva, L. I. (2017). Evaluation of the efficacy of pregabalin in the therapy of chronic pain in patients with knee osteoarthritis. Ter. Arkh. 89, 81-85. doi: 10.17116/terarkh2017891281-85

Finnerup, N. B., Otto, M., Mcquay, H. J., Jensen, T. S., and Sindrup, S. H. (2005). Algorithm for neuropathic pain treatment: an evidence based proposal. Pain 118, 289-305. doi: 10.1016/j.pain.2005.08.013

Fleetwood-Walker, S. M., Hope, P. J., and Mitchell, R. (1988). Antinociceptive actions of descending dopaminergic tracts on cat and rat dorsal horn somatosensory neurones. J. Physiol. 399, 335-348. doi: 10.1113/jphysiol.1988. sp017084

Flores, J. A., El Banoua, F., Galan-Rodriguez, B., and Fernandez-Espejo, E. (2004). Opiate anti-nociception is attenuated following lesion of large dopamine neurons of the periaqueductal grey: critical role for D1 (not D2) dopamine receptors. Pain 110, 205-214. doi: 10.1016/j.pain.2004.03.036

Gao, X., Zhang, Y., and Wu, G. (2001). Effects of dopaminergic agents on carrageenan hyperalgesia after intrathecal administration to rats. Eur. J. Pharmacol. 418, 73-77. doi: 10.1016/s0014-2999(01)00930-x

Ghelardini, C., Galeotti, N., and Bartolini, A. (2000). Antinociception induced by amitriptyline and imipramine is mediated by alpha2A-adrenoceptors. Jpn. J. Pharmacol. 82, 130-137. doi: 10.1254/jjp.82.130

Gilbert, A. K., and Franklin, K. B. (2001). Characterization of the analgesic properties of nomifensine in rats. Pharmacol. Biochem. Behav. 68, 783-787. doi: 10.1016/s0091-3057(01)00479-8

Gilron, I., Bailey, J. M., Tu, D., Holden, R. R., Jackson, A. C., and Houlden, R. L. (2009). Nortriptyline and gabapentin, alone and in combination for neuropathic pain: a double-blind, randomised controlled crossover trial. Lancet 374, 1252-1261. doi: 10.1016/S0140-6736(09)61081-3

Gilron, I., Chaparro, L. E., Tu, D., Holden, R. R., Milev, R., Towheed, T., et al. (2016). Combination of pregabalin with duloxetine for fibromyalgia: a randomized controlled trial. Pain 157, 1532-1540. doi: 10.1097/j.pain. 0000000000000558

Gilron, I., Tu, D., Holden, R. R., Jackson, A. C., and Dumerton-Shore, D. (2015). Combination of morphine with nortriptyline for neuropathic pain. Pain 156, 1440-1448. doi: 10.1097/j.pain.0000000000000149

Glaum, S. R., Proudfit, H. K., and Anderson, E. G. (1988). Reversal of the antinociceptive effects of intrathecally administered serotonin in the rat by a selective 5-HT3 receptor antagonist. Neurosci. Lett. 95, 313-317. doi: 10.1016/ 0304-3940(88)90677-5

Glaum, S. R., Proudfit, H. K., and Anderson, E. G. (1990). 5-HT3 receptors modulate spinal nociceptive reflexes. Brain Res. 510, 12-16. doi: 10.1016/00068993(90)90721-m

Godinez-Chaparro, B., Barragan-Iglesias, P., Castaneda-Corral, G., RochaGonzalez, H. I., and Granados-Soto, V. (2011). Role of peripheral 5-HT(4), 5-HT(6), and 5-HT(7) receptors in development and maintenance of secondary mechanical allodynia and hyperalgesia. Pain 152, 687-697. doi: 10.1016/j.pain. 2010.12.020

Goodman, C. W., and Brett, A. S. (2019). A clinical overview of off-label use of gabapentinoid drugs. JAMA Intern. Med. 179, 695-701. doi: 10.1001/ jamainternmed.2019.0086

Grabow, T. S., Hurley, R. W., Banfor, P. N., and Hammond, D. L. (1999). Supraspinal and spinal delta(2) opioid receptor-mediated antinociceptive synergy is mediated by spinal alpha(2) adrenoceptors. Pain $83,47-55$. doi: 10.1016/s0304-3959(99)00084-6

Gray, A. M., Pache, D. M., and Sewell, R. D. (1999). Do alpha2-adrenoceptors play an integral role in the antinociceptive mechanism of action of antidepressant compounds? Eur. J. Pharmacol. 378, 161-168. doi: 10.1016/s0014-2999(99) 00464- 1
Green, G. M., Scarth, J., and Dickenson, A. (2000). An excitatory role for 5HT in spinal inflammatory nociceptive transmission; state-dependent actions via dorsal horn 5-HT(3) receptors in the anaesthetized rat. Pain 89, 81-88. doi: 10.1016/s0304-3959(00)00346-8

Guiard, B. P., El Mansari, M., and Blier, P. (2008). Cross-talk between dopaminergic and noradrenergic systems in the rat ventral tegmental area, locus ceruleus, and dorsal hippocampus. Mol. Pharmacol. 74, 1463-1475. doi: 10.1124/mol.108.048033

Hache, G., Coudore, F., Gardier, A. M., and Guiard, B. P. (2011). Monoaminergic antidepressants in the relief of pain: potential therapeutic utility of triple reuptake inhibitors (TRIs). Pharmaceuticals 4, 285-342. doi: 10.3390/ ph4020285

Hains, B. C., Everhart, A. W., Fullwood, S. D., and Hulsebosch, C. E. (2002). Changes in serotonin, serotonin transporter expression and serotonin denervation supersensitivity: involvement in chronic central pain after spinal hemisection in the rat. Exp. Neurol. 175, 347-362. doi: 10.1006/exnr.2002.7892

Hajhashemi, V., Banafshe, H. R., Minaiyan, M., Mesdaghinia, A., and Abed, A. (2014). Antinociceptive effects of venlafaxine in a rat model of peripheral neuropathy: role of alpha2-adrenergic receptors. Eur. J. Pharmacol. 738, 230236. doi: 10.1016/j.ejphar.2014.04.046

Hayashi, R., Xiao, W., Kawamoto, M., Yuge, O., and Bennett, G. J. (2008). Systemic glucocorticoid therapy reduces pain and the number of endoneurial tumor necrosis factor-alpha (TNFalpha)-positive mast cells in rats with a painful peripheral neuropathy. J. Pharmacol. Sci. 106, 559-565. doi: 10.1254/jphs. fp0072181

Hayashida, K., Obata, H., Nakajima, K., and Eisenach, J. C. (2008). Gabapentin acts within the locus coeruleus to alleviate neuropathic pain. Anesthesiology 109, 1077-1084. doi: 10.1097/ALN.0b013e31818dac9c

Heinricher, M. M., Morgan, M. M., Tortorici, V., and Fields, H. L. (1994). Disinhibition of off-cells and antinociception produced by an opioid action within the rostral ventromedial medulla. Neuroscience 63, 279-288. doi: 10. 1016/0306-4522(94)90022-1

Heinricher, M. M., Tavares, I., Leith, J. L., and Lumb, B. M. (2009). Descending control of nociception: specificity, recruitment and plasticity. Brain Res. Rev. 60, 214-225. doi: 10.1016/j.brainresrev.2008.12.009

Hernandez, A., Constandil, L., Laurido, C., Pelissier, T., Marchand, F., Ardid, D., et al. (2004). Venlafaxine-induced depression of wind-up activity in mononeuropathic rats is potentiated by inhibition of brain 5-HT1A receptor expression in vivo. Int. J. Neurosci. 114, 229-242. doi: 10.1080/ 00207450490269453

Hirschberg, S., Li, Y., Randall, A., Kremer, E. J., and Pickering, A. E. (2017). Functional dichotomy in spinal- vs prefrontal-projecting locus coeruleus modules splits descending noradrenergic analgesia from ascending aversion and anxiety in rats. eLife 6:e29808. doi: 10.7554/eLife.29808

Hodkinson, D. J., Khawaja, N., O'daly, O., Thacker, M. A., Zelaya, F. O., Wooldridge, C. L., et al. (2015). Cerebral analgesic response to nonsteroidal anti-inflammatory drug ibuprofen. Pain 156, 1301-1310. doi: 10.1097/j.pain. 0000000000000176

Honda, M., Uchida, K., Tanabe, M., and Ono, H. (2006). Fluvoxamine, a selective serotonin reuptake inhibitor, exerts its antiallodynic effects on neuropathic pain in mice via 5-HT2A/2C receptors. Neuropharmacology 51, 866-872. doi: 10.1016/j.neuropharm.2006.05.031

Hoshino, H., Obata, H., Nakajima, K., Mieda, R., and Saito, S. (2015). The antihyperalgesic effects of intrathecal bupropion, a dopamine and noradrenaline reuptake inhibitor, in a rat model of neuropathic pain. Anesth. Analg. 120, 460-466. doi: 10.1213/ANE.00000000000 00540

Hough, L. B., and Rice, F. L. (2011). H3 receptors and pain modulation: peripheral, spinal, and brain interactions. J. Pharmacol. Exp. Ther. 336, 30-37. doi: 10.1124/ jpet.110.171264

Howorth, P. W., Teschemacher, A. G., and Pickering, A. E. (2009). Retrograde adenoviral vector targeting of nociresponsive pontospinal noradrenergic neurons in the rat in vivo. J. Comp. Neurol. 512, 141-157. doi: 10.1002/cne. 21879

Hsieh, G. C., Chandran, P., Salyers, A. K., Pai, M., Zhu, C. Z., Wensink, E. J., et al. (2010). H4 receptor antagonism exhibits anti-nociceptive effects in inflammatory and neuropathic pain models in rats. Pharmacol. Biochem. Behav. 95, 41-50. doi: 10.1016/j.pbb.2009.12.004 
Hughes, S. W., Hickey, L., Hulse, R. P., Lumb, B. M., and Pickering, A. E. (2013). Endogenous analgesic action of the pontospinal noradrenergic system spatially restricts and temporally delays the progression of neuropathic pain following tibial nerve injury. Pain 154, 1680-1690. doi: 10.1016/j.pain.2013. 05.010

Hwang, A. S., and Wilcox, G. L. (1987). Analgesic properties of intrathecally administered heterocyclic antidepressants. Pain 28, 343-355. doi: 10.1016/ 0304-3959(87)90068-6

Ito, S., Suto, T., Saito, S., and Obata, H. (2018). Repeated administration of duloxetine suppresses neuropathic pain by accumulating effects of noradrenaline in the spinal cord. Anesth. Analg. 126, 298-307. doi: 10.1213/ ANE.0000000000002380

Jaggi, A. S., Kaur, G., Bali, A., and Singh, N. (2017). Pharmacological investigations on mast cell stabilizer and histamine receptor antagonists in vincristine-induced neuropathic pain. Naunyn Schmiedebergs Arch. Pharmacol. 390, 1087-1096. doi: 10.1007/s00210-017-1426-8

Jensen, T. S., and Yaksh, T. L. (1989). Comparison of the antinociceptive effect of morphine and glutamate at coincidental sites in the periaqueductal gray and medial medulla in rats. Brain Res. 476, 1-9. doi: 10.1016/0006-8993(89)91529-1

Johansson, F., and Von Knorring, L. (1979). A double-blind controlled study of seratonin uptake inhibitor (Zimelidine) versus placebo in chronic pain patients. Pain 7, 69-78. doi: 10.1016/0304-3959(79)90108-8

Jones, K. L., Finn, D. P., Governo, R. J., Prior, M. J., Morris, P. G., Kendall, D. A., et al. (2009). Identification of discrete sites of action of chronic treatment with desipramine in a model of neuropathic pain. Neuropharmacology 56, 405-413. doi: 10.1016/j.neuropharm.2008.09.010

Kajihara, Y., Murakami, M., Imagawa, T., Otsuguro, K., Ito, S., and Ohta, T. (2010). Histamine potentiates acid-induced responses mediating transient receptor potential V1 in mouse primary sensory neurons. Neuroscience 166, 292-304. doi: 10.1016/j.neuroscience.2009.12.001

Kashiba, H., Fukui, H., and Senba, E. (2001). Histamine H1 receptor mRNA is expressed in capsaicin-insensitive sensory neurons with neuropeptide Y-immunoreactivity in guinea pigs. Brain Res. 901, 85-93. doi: 10.1016/s00068993(01)02287-9

Kato, T., Ide, S., and Minami, M. (2016). Pain relief induces dopamine release in the rat nucleus accumbens during the early but not late phase of neuropathic pain. Neurosci. Lett. 629, 73-78. doi: 10.1016/j.neulet.2016.06.060

Kaur, G., Singh, N., and Jaggi, A. S. (2017). Mast cells in neuropathic pain: an increasing spectrum of their involvement in pathophysiology. Rev. Neurosci. 28, 759-766. doi: 10.1515/revneuro-2017-0007

Kaur, S., Pandhi, P., and Dutta, P. (2011). Painful diabetic neuropathy: an update. Ann. Neurosci. 18, 168-175. doi: 10.5214/ans.0972-7531.1118409

Kellstein, D. E., Malseed, R. T., and Goldstein, F. J. (1984). Contrasting effects of acute vs. chronic tricyclic antidepressant treatment on central morphine analgesia. Pain 20, 323-334. doi: 10.1016/0304-3959(84)90110-6

Kellstein, D. E., Malseed, R. T., and Goldstein, F. J. (1988). Opioid-monoamine interactions in spinal antinociception: evidence for serotonin but not norepinephrine reciprocity. Pain 34, 85-92. doi: 10.1016/0304-3959(88)90 185-6

Kesim, M., Duman, E. N., Kadioglu, M., Yaris, E., Kalyoncu, N. I., and Erciyes, N. (2005). The different roles of 5-HT(2) and 5-HT(3) receptors on antinociceptive effect of paroxetine in chemical stimuli in mice. J. Pharmacol. Sci. 97, 61-66. doi: 10.1254/jphs.fp0040153

Khalilzadeh, E., Azarpey, F., Hazrati, R., and Vafaei Saiah, G. (2018). Evaluation of different classes of histamine $\mathrm{H} 1$ and $\mathrm{H} 2$ receptor antagonist effects on neuropathic nociceptive behavior following tibial nerve transection in rats. Eur. J. Pharmacol. 834, 221-229. doi: 10.1016/j.ejphar.2018.07.011

Khasar, S. G., Mccarter, G., and Levine, J. D. (1999). Epinephrine produces a beta-adrenergic receptor-mediated mechanical hyperalgesia and in vitro sensitization of rat nociceptors. J. Neurophysiol. 81, 1104-1112. doi: 10.1152/ jn.1999.81.3.1104

Kieburtz, K., Simpson, D., Yiannoutsos, C., Max, M. B., Hall, C. D., Ellis, R. J., et al. (1998). A randomized trial of amitriptyline and mexiletine for painful neuropathy in HIV infection. AIDS clinical trial group 242 protocol team. Neurology 51, 1682-1688. doi: 10.1212/wnl.51.6.1682

Kim, D. S., Choi, J. O., Rim, H. D., and Cho, H. J. (2002). Downregulation of voltage-gated potassium channel alpha gene expression in dorsal root ganglia following chronic constriction injury of the rat sciatic nerve. Brain Res. Mol. Brain Res. 105, 146-152. doi: 10.1016/s0169-328x(02)00388-1

Kim, D. S., Lee, S. J., Park, S. Y., Yoo, H. J., Kim, S. H., Kim, K. J., et al. (2001). Differentially expressed genes in rat dorsal root ganglia following peripheral nerve injury. Neuroreport 12, 3401-3405. doi: 10.1097/00001756-20011029000050

Kim, J. Y., Tillu, D. V., Quinn, T. L., Mejia, G. L., Shy, A., Asiedu, M. N., et al. (2015). Spinal dopaminergic projections control the transition to pathological pain plasticity via a D1/D5-mediated mechanism. J. Neurosci. 35, 6307-6317. doi: 10.1523/JNEUROSCI.3481-14.2015

King, J. B., Schauerhamer, M. B., and Bellows, B. K. (2015). A review of the clinical utility of duloxetine in the treatment of diabetic peripheral neuropathic pain. Ther. Clin. Risk Manag. 11, 1163-1175. doi: 10.2147/TCRM.S74165

Kishimoto, K., Koyama, S., and Akaike, N. (2001). Synergistic mu-opioid and 5 -HT1A presynaptic inhibition of GABA release in rat periaqueductal gray neurons. Neuropharmacology 41, 529-538. doi: 10.1016/s0028-3908(01)00 $100-9$

Kremer, M., Yalcin, I., Goumon, Y., Wurtz, X., Nexon, L., Daniel, D., et al. (2018). A dual noradrenergic mechanism for the relief of neuropathic allodynia by the antidepressant drugs duloxetine and amitriptyline. J. Neurosci. 38, 9934-9954. doi: 10.1523/JNEUROSCI.1004-18.2018

Kupers, R., Frokjaer, V. G., Naert, A., Christensen, R., Budtz-Joergensen, E., Kehlet, H., et al. (2009). A PET [18F]altanserin study of 5-HT2A receptor binding in the human brain and responses to painful heat stimulation. Neuroimage 44, 1001-1007. doi: 10.1016/j.neuroimage.2008.10.011

Lang, P. M., Moalem-Taylor, G., Tracey, D. J., Bostock, H., and Grafe, P. (2006). Activity-dependent modulation of axonal excitability in unmyelinated peripheral rat nerve fibers by the 5-HT(3) serotonin receptor. J. Neurophysiol. 96, 2963-2971. doi: 10.1152/jn.00716.2006

Lashbrook, J. M., Ossipov, M. H., Hunter, J. C., Raffa, R. B., Tallarida, R. J., and Porreca, F. (1999). Synergistic antiallodynic effects of spinal morphine with ketorolac and selective COX1- and COX2-inhibitors in nerve-injured rats. Pain 82, 65-72. doi: 10.1016/s0304-3959(99)00031-7

Lima, D., and Almeida, A. (2002). The medullary dorsal reticular nucleus as a pronociceptive centre of the pain control system. Prog. Neurobiol. 66, 81-108. doi: 10.1016/s0301-0082(01)00025-9

Lischetzki, G., Rukwied, R., Handwerker, H. O., and Schmelz, M. (2001). Nociceptor activation and protein extravasation induced by inflammatory mediators in human skin. Eur. J. Pain 5, 49-57. doi: 10.1053/eujp.2000.0214

Llorca-Torralba, M., Borges, G., Neto, F., Mico, J. A., and Berrocoso, E. (2016). Noradrenergic locus coeruleus pathways in pain modulation. Neuroscience 338, 93-113. doi: 10.1016/j.neuroscience.2016.05.057

Llorca-Torralba, M., Suarez-Pereira, I., Bravo, L., Camarena-Delgado, C., GarciaPartida, J. A., Mico, J. A., et al. (2019). Chemogenetic silencing of the locus coeruleus-basolateral amygdala pathway abolishes pain-induced anxiety and enhanced aversive learning in rats. Biol. Psychiatry 85, 1021-1035. doi: 10.1016/ j.biopsych.2019.02.018

Lopez-Canul, M., Comai, S., Dominguez-Lopez, S., Granados-Soto, V., and Gobbi, G. (2015). Antinociceptive properties of selective MT(2) melatonin receptor partial agonists. Eur. J. Pharmacol. 764, 424-432. doi: 10.1016/j.ejphar.2015. 07.010

Loughlin, S. E., Foote, S. L., and Grzanna, R. (1986). Efferent projections of nucleus locus coeruleus: morphologic subpopulations have different efferent targets. Neuroscience 18, 307-319. doi: 10.1016/0306-4522(86)90156-9

Luo, L., Puke, M. J., and Wiesenfeld-Hallin, Z. (1994). The effects of intrathecal morphine and clonidine on the prevention and reversal of spinal cord hyperexcitability following sciatic nerve section in the rat. Pain 58, 245-252. doi: 10.1016/0304-3959(94)90205-4

Magnusson, J. E., and Fisher, K. (2000). The involvement of dopamine in nociception: the role of $\mathrm{D}(1)$ and $\mathrm{D}(2)$ receptors in the dorsolateral striatum. Brain Res. 855, 260-266. doi: 10.1016/s0006-8993(99)02396-3

Malmberg, K. J., Arulampalam, V., Ichihara, F., Petersson, M., Seki, K., Andersson, $\mathrm{T}$., et al. (2001). Inhibition of activated/memory $(\mathrm{CD} 45 \mathrm{RO}(+)) \mathrm{T}$ cells by oxidative stress associated with block of NF-kappaB activation. J. Immunol. 167, 2595-2601. doi: 10.4049/jimmunol.167.5.2595

Marchand, F., Alloui, A., Chapuy, E., Hernandez, A., Pelissier, T., Ardid, D., et al. (2003). The antihyperalgesic effect of venlafaxine in diabetic rats does not 
involve the opioid system. Neurosci. Lett. 342, 105-108. doi: 10.1016/s03043940(03)00270-2

Martins, I., Carvalho, P., De Vries, M. G., Teixeira-Pinto, A., Wilson, S. P., Westerink, B. H., et al. (2015). Increased noradrenergic neurotransmission to a pain facilitatory area of the brain is implicated in facilitation of chronic pain. Anesthesiology 123, 642-653. doi: 10.1097/ALN.0000000000000749

Maruo, K., Yamamoto, H., Yamamoto, S., Nagata, T., Fujikawa, H., Kanno, T., et al. (2006). Modulation of $\mathrm{P} 2 \mathrm{X}$ receptors via adrenergic pathways in rat dorsal root ganglion neurons after sciatic nerve injury. Pain 120, 106-112. doi: 10.1016/j.pain.2005.10.016

Max, M. B., Kishore-Kumar, R., Schafer, S. C., Meister, B., Gracely, R. H., Smoller, B., et al. (1991). Efficacy of desipramine in painful diabetic neuropathy: a placebo-controlled trial. Pain 45, 3-9; discussion 1-2.

Max, M. B., Lynch, S. A., Muir, J., Shoaf, S. E., Smoller, B., and Dubner, R. (1992). Effects of desipramine, amitriptyline, and fluoxetine on pain in diabetic neuropathy. N. Engl. J. Med. 326, 1250-1256. doi: 10.1056/ nejm199205073261904

McCleane, G. J., Suzuki, R., and Dickenson, A. H. (2003). Does a single intravenous injection of the $5 \mathrm{HT} 3$ receptor antagonist ondansetron have an analgesic effect in neuropathic pain? A double-blinded, placebo-controlled cross-over study. Anesth. Analg. 97, 1474-1478. doi: 10.1213/01.ane.0000085640.69855.51

McGaraughty, S., Chu, K. L., Cowart, M. D., and Brioni, J. D. (2012). Antagonism of supraspinal histamine $\mathrm{H} 3$ receptors modulates spinal neuronal activity in neuropathic rats. J. Pharmacol. Exp. Ther. 343, 13-20. doi: 10.1124/jpet.112. 194761

McLachlan, E. M., Janig, W., Devor, M., and Michaelis, M. (1993). Peripheral nerve injury triggers noradrenergic sprouting within dorsal root ganglia. Nature 363, 543-546. doi: 10.1038/363543a0

Meske, D. S., Xie, J. Y., Oyarzo, J., Badghisi, H., Ossipov, M. H., and Porreca, F. (2014). Opioid and noradrenergic contributions of tapentadol in experimental neuropathic pain. Neurosci. Lett. 562, 91-96. doi: 10.1016/j.neulet.2013.08.017

Meyer, P. J., Morgan, M. M., Kozell, L. B., and Ingram, S. L. (2009). Contribution of dopamine receptors to periaqueductal gray-mediated antinociception. Psychopharmacology 204, 531-540. doi: 10.1007/s00213-009-1482-y

Michaelis, M., and Janig, W. (1998). Sympathetic nervous system and pain: pathophysiological mechanisms. Schmerz 12, 261-271.

Mico, J. A., Ardid, D., Berrocoso, E., and Eschalier, A. (2006). Antidepressants and pain. Trends Pharmacol. Sci. 27, 348-354.

Mico, J. A., Gibert-Rahola, J., Casas, J., Rojas, O., Serrano, M. I., and Serrano, J. S. (1997). Implication of beta 1 - and beta 2-adrenergic receptors in the antinociceptive effect of tricyclic antidepressants. Eur. Neuropsychopharmacol. 7, 139-145. doi: 10.1016/s0924-977x(97)00411-2

Millan, M. J. (2002). Descending control of pain. Prog. Neurobiol. 66, 355-474.

Mojtahedin, A. (2016). Effects of cholinergic system in antinociception induced by $\mathrm{H}-1$ and $\mathrm{H}-2$ receptor antagonists on somatic pain in rats. Int. J. Med. Res. Health Sci. 5, 378-383.

Moore, R. A., Derry, S., Aldington, D., Cole, P., and Wiffen, P. J. (2015). Amitriptyline for neuropathic pain in adults. Cochrane Database Syst. Rev. 7:CD008242.

Morello, C. M., Leckband, S. G., Stoner, C. P., Moorhouse, D. F., and Sahagian, G. A. (1999). Randomized double-blind study comparing the efficacy of gabapentin with amitriptyline on diabetic peripheral neuropathy pain. Arch. Intern. Med. 159, 1931-1937.

Morgado, C., Silva, L., Pereira-Terra, P., and Tavares, I. (2011). Changes in serotoninergic and noradrenergic descending pain pathways during painful diabetic neuropathy: the preventive action of IGF1. Neurobiol. Dis. 43, 275-284. doi: 10.1016/j.nbd.2011.04.001

Moron, J. A., Brockington, A., Wise, R. A., Rocha, B. A., and Hope, B. T. (2002). Dopamine uptake through the norepinephrine transporter in brain regions with low levels of the dopamine transporter: evidence from knockout mouse lines. J. Neurosci. 22, 389-395. doi: 10.1523/jneurosci.22-02-00389. 2002

Nakajima, K., Obata, H., Ito, N., Goto, F., and Saito, S. (2009). The nociceptive mechanism of 5-hydroxytryptamine released into the peripheral tissue in acute inflammatory pain in rats. Eur. J. Pain 13, 441-447. doi: 10.1016/j.ejpain.2008. 06.007

Nakamura, M., and Ferreira, S. H. (1988). Peripheral analgesic action of clonidine: mediation by release of endogenous enkephalin-like substances. Eur. J. Pharmacol. 146, 223-228. doi: 10.1016/0014-2999(88)90 296-8

Navratilova, E., Xie, J. Y., Okun, A., Qu, C., Eyde, N., Ci, S., et al. (2012). Pain relief produces negative reinforcement through activation of mesolimbic rewardvaluation circuitry. Proc. Natl. Acad. Sci. U.S.A. 109, 20709-20713. doi: 10.1073/ pnas. 1214605109

Neubert, M. J., Kincaid, W., and Heinricher, M. M. (2004). Nociceptive facilitating neurons in the rostral ventromedial medulla. Pain 110, 158-165. doi: 10.1016/ j.pain.2004.03.017

Oatway, M. A., Chen, Y., and Weaver, L. C. (2004). The 5-HT3 receptor facilitates at-level mechanical allodynia following spinal cord injury. Pain 110, 259-268. doi: 10.1016/j.pain.2004.03.040

Obara, I., Telezhkin, V., Alrashdi, I., and Chazot, P. L. (2019). Histamine, histamine receptors, and neuropathic pain relief. Br. J. Pharmacol. doi: 10.1111/bph.14696 [Epub ahead of print].

Obata, H. (2017). Analgesic mechanisms of antidepressants for neuropathic pain. Int. J. Mol. Sci. 18:2483. doi: 10.3390/ijms18112483

Obata, H., Saito, S., Sasaki, M., Ishizaki, K., and Goto, F. (2001). Antiallodynic effect of intrathecally administered 5-HT(2) agonists in rats with nerve ligation. Pain 90, 173-179. doi: 10.1016/s0304-3959(00)00401-2

Oliveras, J. L., Redjemi, F., Guilbaud, G., and Besson, J. M. (1975). Analgesia induced by electrical stimulation of the inferior centralis nucleus of the raphe in the cat. Pain 1, 139-145. doi: 10.1016/0304-3959(75)90098-6

Ong, C. K., Lirk, P., Tan, C. H., and Seymour, R. A. (2007). An evidence-based update on nonsteroidal anti-inflammatory drugs. Clin. Med. Res. 5, 19-34. doi: $10.3121 / \mathrm{cmr} .2007 .698$

Ozdogan, U. K., Lahdesmaki, J., Mansikka, H., and Scheinin, M. (2004). Loss of amitriptyline analgesia in alpha 2A-adrenoceptor deficient mice. Eur. J. Pharmacol. 485, 193-196. doi: 10.1016/j.ejphar.2003. 11.047

Panula, P., Chazot, P. L., Cowart, M., Gutzmer, R., Leurs, R., Liu, W. L., et al. (2015). International union of basic and clinical pharmacology. XCVIII. Histamine receptors. Pharmacol. Rev. 67, 601-655. doi: 10.1124/pr.114.01 0249

Paqueron, X., Leguen, M., Rosenthal, D., Coriat, P., Willer, J. C., and Danziger, N. (2003). The phenomenology of body image distortions induced by regional anaesthesia. Brain 126, 702-712. doi: 10.1093/brain/awg063

Parada, C. A., Yeh, J. J., Joseph, E. K., and Levine, J. D. (2003). Tumor necrosis factor receptor type-1 in sensory neurons contributes to induction of chronic enhancement of inflammatory hyperalgesia in rat. Eur. J. Neurosci. 17, 1847-1852. doi: 10.1046/j.1460-9568.2003.02626.x

Parsons, M. E., and Ganellin, C. R. (2006). Histamine and its receptors. Br. J. Pharmacol. 147(Suppl. 1), S127-S135.

Paulus, W., and Trenkwalder, C. (2006). Less is more: pathophysiology of dopaminergic-therapy-related augmentation in restless legs syndrome. Lancet Neurol. 5, 878-886. doi: 10.1016/s1474-4422(06)70576-2

Perrot, S., Javier, R. M., Marty, M., Le Jeunne, C., Laroche, F., CEDR, et al. (2008). Is there any evidence to support the use of anti-depressants in painful rheumatological conditions? Systematic review of pharmacological and clinical studies. Rheumatology 47, 1117-1123. doi: 10.1093/rheumatology/ ken 110

Perrot, S., Maheu, E., Javier, R. M., Eschalier, A., Coutaux, A., LeBars, M., et al. (2006). Guidelines for the use of antidepressants in painful rheumatic conditions. Eur. J. Pain 10, 185-192.

Pertovaara, A. (2006). Noradrenergic pain modulation. Prog. Neurobiol. 80, 53-83. doi: 10.1016/j.pneurobio.2006.08.001

Peters, C. M., Hayashida, K., Ewan, E. E., Nakajima, K., Obata, H., Xu, Q., et al. (2010). Lack of analgesic efficacy of spinal ondansetron on thermal and mechanical hypersensitivity following spinal nerve ligation in the rat. Brain Res. 1352, 83-93. doi: 10.1016/j.brainres.2010.07.020

Pichon, X., Wattiez, A. S., Becamel, C., Ehrlich, I., Bockaert, J., Eschalier, A., et al. (2010). Disrupting 5-HT(2A) receptor/PDZ protein interactions reduces hyperalgesia and enhances SSRI efficacy in neuropathic pain. Mol. Ther. 18, 1462-1470. doi: 10.1038/mt.2010.101

Pierce, P. A., Xie, G. X., Levine, J. D., and Peroutka, S. J. (1996). 5Hydroxytryptamine receptor subtype messenger RNAs in rat peripheral sensory and sympathetic ganglia: a polymerase chain reaction study. Neuroscience 70 , 553-559. doi: 10.1016/0306-4522(95)00329-0 
Pini, L. A., Sandrini, M., and Vitale, G. (1996). The antinociceptive action of paracetamol is associated with changes in the serotonergic system in the rat brain. Eur. J. Pharmacol. 308, 31-40. doi: 10.1016/0014-2999(96)00261-0

Popiolek-Barczyk, K., Lazewska, D., Latacz, G., Olejarz, A., Makuch, W., Stark, H., et al. (2018). Antinociceptive effects of novel histamine H3 and H4 receptor antagonists and their influence on morphine analgesia of neuropathic pain in the mouse. Br. J. Pharmacol. 175, 2897-2910. doi: 10.1111/bph.14185

Poree, L. R., Guo, T. Z., Kingery, W. S., and Maze, M. (1998). The analgesic potency of dexmedetomidine is enhanced after nerve injury: a possible role for peripheral alpha2-adrenoceptors. Anesth. Analg. 87, 941-948. doi: 10.1097/ 00000539-199810000-00037

Potvin, S., Grignon, S., and Marchand, S. (2009). Human evidence of a supraspinal modulating role of dopamine on pain perception. Synapse 63, 390-402. doi: $10.1002 /$ syn.20616

Rahman, W., Suzuki, R., Webber, M., Hunt, S. P., and Dickenson, A. H. (2006). Depletion of endogenous spinal 5-HT attenuates the behavioural hypersensitivity to mechanical and cooling stimuli induced by spinal nerve ligation. Pain 123, 264-274. doi: 10.1016/j.pain.2006.02.033

Reimann, W., Schlutz, H., and Selve, N. (1999). The antinociceptive effects of morphine, desipramine, and serotonin and their combinations after intrathecal injection in the rat. Anesth. Analg. 88, 141-145. doi: 10.1097/00000539199901000-00026

Richards, B. L., Whittle, S. L., and Buchbinder, R. (2011). Antidepressants for pain management in rheumatoid arthritis. Cochrane Database Syst. Rev. 11:CD008920. doi: 10.1002/14651858.CD008920.pub2

Robinson, L. R., Czerniecki, J. M., Ehde, D. M., Edwards, W. T., Judish, D. A., Goldberg, M. L., et al. (2004). Trial of amitriptyline for relief of pain in amputees: results of a randomized controlled study. Arch. Phys. Med. Rehabil. $85,1-6$.

Rocha-González, H. I., Meneses, A., Carlton, S. M., and Granados-Soto, V. (2005). Pronociceptive role of peripheral and spinal 5-HT7 receptors in the formalin test. Pain 117, 182-192. doi: 10.1016/j.pain.2005.06.011

Rojas-Corrales, M. O., Berrocoso, E., and Mico, J. A. (2005). Role of 5-HT1A and 5-HT1B receptors in the antinociceptive effect of tramadol. Eur. J. Pharmacol. 511, 21-26. doi: 10.1016/j.ejphar.2005.02.006

Rojas-Corrales, M. O., Ortega-Alvaro, A., Gibert-Rahola, J., Roca-Vinardell, A., and Mico, J. A. (2000). Pindolol, a beta-adrenoceptor blocker/5hydroxytryptamine $(1 \mathrm{~A} / 1 \mathrm{~B})$ antagonist, enhances the analgesic effect of tramadol. Pain 88, 119-124. doi: 10.1016/s0304-3959(00)00299-2

Rojo, M. L., Rodriguez-Gaztelumendi, A., Pazos, A., and Diaz, A. (2012). Differential adaptive changes on serotonin and noradrenaline transporters in a rat model of peripheral neuropathic pain. Neurosci. Lett. 515, 181-186. doi: 10.1016/j.neulet.2012.03.050

Romero, T. R., Guzzo, L. S., and Duarte, I. D. (2012). Mu, delta, and kappa opioid receptor agonists induce peripheral antinociception by activation of endogenous noradrenergic system. J. Neurosci. Res. 90, 1654-1661. doi: 10. 1002/jnr.23050

Ruffolo, R. R. Jr., and Hieble, J. P. (1994). Alpha-adrenoceptors. Pharmacol. Ther. 61, 1-64.

Saarto, T., and Wiffen, P. J. (2007). Antidepressants for neuropathic pain. Cochrane Database Syst. Rev. 4:CD005454.

Sagheddu, C., Aroni, S., De Felice, M., Lecca, S., Luchicchi, A., Melis, M., et al. (2015). Enhanced serotonin and mesolimbic dopamine transmissions in a rat model of neuropathic pain. Neuropharmacology 97, 383-393. doi: 10.1016/j. neuropharm.2015.06.003

Sandrini, M., Ottani, A., Vitale, G., and Pini, L. A. (1998). Acetylsalicylic acid potentiates the antinociceptive effect of morphine in the rat: involvement of the central serotonergic system. Eur. J. Pharmacol. 355, 133-140. doi: 10.1016/ s0014-2999(98)00496-8

Sanna, M. D., Lucarini, L., Durante, M., Ghelardini, C., Masini, E., and Galeotti, N. (2017). Histamine $\mathrm{H}_{4}$ receptor agonist-induced relief from painful peripheral neuropathy is mediated by inhibition of spinal neuroinflammation and oxidative stress. Br. J. Pharmacol. 174, 28-40. doi: 10.1111/bph. 13644

Sanna, M. D., Stark, H., Lucarini, L., Ghelardini, C., Masini, E., and Galeotti, N. (2015). Histamine H4 receptor activation alleviates neuropathic pain through differential regulation of ERK, JNK, and P38 MAPK phosphorylation. Pain 156, 2492-2504. doi: 10.1097/j.pain.0000000000000319
Saper, J. R., Silberstein, S. D., Lake, A. E. III, and Winters, M. E. (1994). Doubleblind trial of fluoxetine: chronic daily headache and migraine. Headache 34, 497-502. doi: 10.1111/j.1526-4610.1994.hed3409497.x

Saper, J. R., Silberstein, S. D., Lake, A. E. III, and Winters, M. E. (1995). Fluoxetine and migraine: comparison of double-blind trials. Headache 35:233. doi: 10 . 1111/j.1526-4610.1995.hed3504233_1.x

Sara, S. J., and Bouret, S. (2012). Orienting and reorienting: the locus coeruleus mediates cognition through arousal. Neuron 76, 130-141. doi: 10.1016/j. neuron.2012.09.011

Sato, J., and Perl, E. R. (1991). Adrenergic excitation of cutaneous pain receptors induced by peripheral nerve injury. Science 251, 1608-1610. doi: 10.1126/ science. 2011742

Satoh, M., Oku, R., and Akaike, A. (1983). Analgesia produced by microinjection of L-glutamate into the rostral ventromedial bulbar nuclei of the rat and its inhibition by intrathecal alpha-adrenergic blocking agents. Brain Res. 261, 361-364. doi: 10.1016/0006-8993(83)90646-7

Sawynok, J., Esser, M. J., and Reid, A. R. (2001). Antidepressants as analgesics: an overview of central and peripheral mechanisms of action. J. Psychiatry Neurosci. 26, 21-29.

Schroder, W., Tzschentke, T. M., Terlinden, R., De Vry, J., Jahnel, U., Christoph, T., et al. (2011). Synergistic interaction between the two mechanisms of action of tapentadol in analgesia. J. Pharmacol. Exp. Ther. 337, 312-320. doi: 10.1124/ jpet.110.175042

Scott, D. J., Heitzeg, M. M., Koeppe, R. A., Stohler, C. S., and Zubieta, J. K. (2006). Variations in the human pain stress experience mediated by ventral and dorsal basal ganglia dopamine activity. J. Neurosci. 26, 10789-10795. doi: 10.1523/jneurosci.2577-06.2006

Semenchuk, M. R., and Davis, B. (2000). Efficacy of sustained-release bupropion in neuropathic pain: an open-label study. Clin. J. Pain 16, 6-11. doi: 10.1097/ 00002508-200003000-00002

Shea, V. K., and Perl, E. R. (1985). Failure of sympathetic stimulation to affect responsiveness of rabbit polymodal nociceptors. J. Neurophysiol. 54, 513-519. doi: 10.1152/jn.1985.54.3.513

Shen, F., Tsuruda, P. R., Smith, J. A., Obedencio, G. P., and Martin, W. J. (2013). Relative contributions of norepinephrine and serotonin transporters to antinociceptive synergy between monoamine reuptake inhibitors and morphine in the rat formalin model. PLoS One 8:e74891. doi: 10.1371/journal. pone.0074891

Shi, T. S., Winzer-Serhan, U., Leslie, F., and Hokfelt, T. (2000). Distribution and regulation of alpha(2)-adrenoceptors in rat dorsal root ganglia. Pain 84, 319-330. doi: 10.1016/s0304-3959(99)00224-9

Silva, L. C., Miranda, E., Castor, M. G., Souza, T. C., Duarte, I. D., and Romero, T. R. (2015). NSAIDs induce peripheral antinociception by interaction with the adrenergic system. Life Sci. 130, 7-11. doi: 10.1016/j.lfs.2015. 03.011

Silverfield, J. C., Kamin, M., Wu, S. C., Rosenthal, N., and Group, C.-S. (2002). Tramadol/acetaminophen combination tablets for the treatment of osteoarthritis flare pain: a multicenter, outpatient, randomized, double-blind, placebo-controlled, parallel-group, add-on study. Clin. Ther. 24, 282-297. doi: 10.1016/s0149-2918(02)85024-x

Sindrup, S. H., Bjerre, U., Dejgaard, A., Brosen, K., Aaes-Jorgensen, T., and Gram, L. F. (1992). The selective serotonin reuptake inhibitor citalopram relieves the symptoms of diabetic neuropathy. Clin. Pharmacol. Ther. 52, 547-552. doi: 10.1038/clpt.1992.183

Sindrup, S. H., Gram, L. F., Brosen, K., Eshoj, O., and Mogensen, E. F. (1990). The selective serotonin reuptake inhibitor paroxetine is effective in the treatment of diabetic neuropathy symptoms. Pain 42, 135-144. doi: 10.1016/0304-3959(90) 91157-e

Sindrup, S. H., and Jensen, T. S. (2000). Pharmacologic treatment of pain in polyneuropathy. Neurology 55, 915-920. doi: 10.1212/wnl.55.7.915

Sindrup, S. H., Otto, M., Finnerup, N. B., and Jensen, T. S. (2005). Antidepressants in the treatment of neuropathic pain. Basic Clin. Pharmacol. Toxicol. 96, 399-409.

Singh, V. P., Jain, N. K., and Kulkarni, S. K. (2001). On the antinociceptive effect of fluoxetine, a selective serotonin reuptake inhibitor. Brain Res. 915, 218-226. doi: 10.1016/s0006-8993(01)02854-2

Sounvoravong, S., Nakashima, M. N., Wada, M., and Nakashima, K. (2004). Decrease in serotonin concentration in raphe magnus nucleus and attenuation 
of morphine analgesia in two mice models of neuropathic pain. Eur. J. Pharmacol. 484, 217-223. doi: 10.1016/j.ejphar.2003.11.028

Sprouse, J. S., and Aghajanian, G. K. (1987). Electrophysiological responses of serotoninergic dorsal raphe neurons to 5-HT1A and 5-HT1B agonists. Synapse 1, 3-9. doi: 10.1002/syn.890010103

Stone, L. S., Vulchanova, L., Riedl, M. S., Wang, J., Williams, F. G., Wilcox, G. L., et al. (1999). Effects of peripheral nerve injury on alpha-2A and alpha-2C adrenergic receptor immunoreactivity in the rat spinal cord. Neuroscience 93, 1399-1407. doi: 10.1016/s0306-4522(99)00209-2

Stratz, T., and Muller, W. (2000). The use of 5-HT3 receptor antagonists in various rheumatic diseases-a clue to the mechanism of action of these agents in fibromyalgia? Scand. J. Rheumatol. Suppl. 113, 66-71. doi: 10.1080/ 030097400446689

Suzuki, R., Rygh, L. J., and Dickenson, A. H. (2004). Bad news from the brain: descending 5-HT pathways that control spinal pain processing. Trends Pharmacol. Sci. 25, 613-617. doi: 10.1016/j.tips.2004.10.002

Taiwo, Y. O., Heller, P. H., and Levine, J. D. (1992). Mediation of serotonin hyperalgesia by the cAMP second messenger system. Neuroscience 48, 479-483. doi: 10.1016/0306-4522(92)90507-x

Takasu, K., Honda, M., Ono, H., and Tanabe, M. (2006). Spinal alpha(2)-adrenergic and muscarinic receptors and the NO release cascade mediate supraspinally produced effectiveness of gabapentin at decreasing mechanical hypersensitivity in mice after partial nerve injury. Br. J. Pharmacol. 148, 233-244. doi: 10.1038/ sj.bjp.0706731

Takeuchi, Y., Takasu, K., Honda, M., Ono, H., and Tanabe, M. (2007). Neurochemical evidence that supraspinally administered gabapentin activates the descending noradrenergic system after peripheral nerve injury. Eur. J. Pharmacol. 556, 69-74. doi: 10.1016/j.ejphar.2006.10.059

Tamaddonfard, E., and Hamzeh-Gooshchi, N. (2014). Effects of administration of histamine and its $\mathrm{H} 1, \mathrm{H} 2$, and $\mathrm{H} 3$ receptor antagonists into the primary somatosensory cortex on inflammatory pain in rats. Iran J. Basic Med. Sci. 17, 55-61.

Tanabe, M., Takasu, K., Takeuchi, Y., and Ono, H. (2008). Pain relief by gabapentin and pregabalin via supraspinal mechanisms after peripheral nerve injury. J. Neurosci. Res. 86, 3258-3264. doi: 10.1002/jnr.21786

Taylor, B. K., Joshi, C., and Uppal, H. (2003). Stimulation of dopamine D2 receptors in the nucleus accumbens inhibits inflammatory pain. Brain Res. 987, 135-143. doi: 10.1016/s0006-8993(03)03318-3

Tazawa, T., Kamiya, Y., Kobayashi, A., Saeki, K., Takiguchi, M., Nakahashi, Y., et al. (2015). Spinal cord stimulation modulates supraspinal centers of the descending antinociceptive system in rats with unilateral spinal nerve injury. Mol. Pain 11:36. doi: 10.1186/s12990-015-0039-9

Tesfaye, S., Wilhelm, S., Lledo, A., Schacht, A., Tolle, T., Bouhassira, D., et al. (2013). Duloxetine and pregabalin: high-dose monotherapy or their combination? The "COMBO-DN study"-a multinational, randomized, doubleblind, parallel-group study in patients with diabetic peripheral neuropathic pain. Pain 154, 2616-2625. doi: 10.1016/j.pain.2013.05.043

Thibault, K., Van Steenwinckel, J., Brisorgueil, M. J., Fischer, J., Hamon, M., Calvino, B., et al. (2008). Serotonin 5-HT2A receptor involvement and Fos expression at the spinal level in vincristine-induced neuropathy in the rat. Pain 140, 305-322. doi: 10.1016/j.pain.2008.09.006

Tracey, I., and Bushnell, M. C. (2009). How neuroimaging studies have challenged us to rethink: is chronic pain a disease? J. Pain 10, 1113-1120. doi: 10.1016/j. jpain.2009.09.001

Tuveson, B., Leffler, A. S., and Hansson, P. (2011). Ondansetron, a 5HT3antagonist, does not alter dynamic mechanical allodynia or spontaneous ongoing pain in peripheral neuropathy. Clin. J. Pain 27, 323-329. doi: 10.1097/ AJP.0b013e31820215c5

Ücel, U. I., Can, O. D., Demir Ozkay, U., and Ozturk, Y. (2015). Antihyperalgesic and antiallodynic effects of mianserin on diabetic neuropathic pain: a study on mechanism of action. Eur. J. Pharmacol. 756, 92-106. doi: 10.1016/j.ejphar. 2015.02.048

Urban, M. O., and Gebhart, G. F. (1999). Supraspinal contributions to hyperalgesia. Proc. Natl. Acad. Sci. U.S.A. 96, 7687-7692. doi: 10.1073/pnas.96.14. 7687

van den Driest, J. J., Bierma-Zeinstra, S. M. A., Bindels, P. J. E., and Schiphof, D. (2017). Amitriptyline for musculoskeletal complaints: a systematic review. Fam. Pract. 34, 138-146. doi: 10.1093/fampra/cmw134
Van Hecke, O., Torrance, N., and Smith, B. H. (2013). Chronic pain epidemiology and its clinical relevance. Br. J. Anaesth. 111, 13-18. doi: 10.1093/bja/ aet123

Vergne-Salle, P., Dufauret-Lombard, C., Bonnet, C., Simon, A., Treves, R., Bonnabau, H., et al. (2011). A randomised, double-blind, placebo-controlled trial of dolasetron, a 5-hydroxytryptamine 3 receptor antagonist, in patients with fibromyalgia. Eur. J. Pain 15, 509-514. doi: 10.1016/j.ejpain.2010. 09.013

Viguier, F., Michot, B., Hamon, M., and Bourgoin, S. (2013). Multiple roles of serotonin in pain control mechanisms-implications of 5-HT(7) and other 5-HT receptor types. Eur. J. Pharmacol. 716, 8-16. doi: 10.1016/j.ejphar.2013.01.074

Vo, T., Rice, A. S., and Dworkin, R. H. (2009). Non-steroidal anti-inflammatory drugs for neuropathic pain: how do we explain continued widespread use? Pain 143, 169-171. doi: 10.1016/j.pain.2009.03.013

Vogel, C., Mössner, R., Gerlach, M., Heinemann, T., Murphy, D. L., Riederer, P., et al. (2003). Absence of thermal hyperalgesia in serotonin transporter-deficient mice. J. Neurosci. 23, 708-715. doi: 10.1523/jneurosci.23-02-00708.2003

Vrethem, M., Boivie, J., Arnqvist, H., Holmgren, H., Lindstrom, T., and Thorell, L. H. (1997). A comparison a amitriptyline and maprotiline in the treatment of painful polyneuropathy in diabetics and nondiabetics. Clin. J. Pain 13, 313-323. doi: 10.1097/00002508-199712000-00009

Wei, H., and Pertovaara, A. (2013). Regulation of neuropathic hypersensitivity by alpha(2) -adrenoceptors in the pontine A7 cell group. Basic Clin. Pharmacol. Toxicol. 112, 90-95. doi: 10.1111/j.1742-7843.2012.00930.x

Wiffen, P. J., Derry, S., Moore, R. A., Aldington, D., Cole, P., Rice, A. S., et al. (2013). Antiepileptic drugs for neuropathic pain and fibromyalgia - an overview of Cochrane reviews. Cochrane Database Syst. Rev. 11:CD010567. doi: 10.1002/ 14651858.CD010567.pub2

Wigdor, S., and Wilcox, G. L. (1987). Central and systemic morphineinduced antinociception in mice: contribution of descending serotonergic and noradrenergic pathways. J. Pharmacol. Exp. Ther. 242, 90-95.

Wu, S., Zhu, M., Wang, W., Wang, Y., Li, Y., and Yew, D. T. (2001). Changes of the expression of 5-HT receptor subtype mRNAs in rat dorsal root ganglion by complete Freund's adjuvant-induced inflammation. Neurosci. Lett. 307, 183-186. doi: 10.1016/s0304-3940(01)01946-2

Xie, J., Ho Lee, Y., Wang, C., Mo Chung, J., and Chung, K. (2001). Differential expression of alpha1-adrenoceptor subtype mRNAs in the dorsal root ganglion after spinal nerve ligation. Brain Res. Mol. Brain Res. 93, 164-172. doi: 10.1016/ s0169-328x(01)00201-7

Yalcin, I., Tessier, L. H., Petit-Demouliere, N., Doridot, S., Hein, L., FreundMercier, M. J., et al. (2009). Beta2-adrenoceptors are essential for desipramine, venlafaxine or reboxetine action in neuropathic pain. Neurobiol. Dis. 33, 386394. doi: 10.1016/j.nbd.2008.11.003

Yalcin, I., Tessier, L. H., Petit-Demouliere, N., Waltisperger, E., Hein, L., FreundMercier, M. J., et al. (2010). Chronic treatment with agonists of beta(2)adrenergic receptors in neuropathic pain. Exp. Neurol. 221, 115-121. doi: 10. 1016/j.expneurol.2009.10.008

Yang, H. W., Zhou, L. J., Hu, N. W., Xin, W. J., and Liu, X. G. (2005). Activation of spinal $\mathrm{d} 1 / \mathrm{d} 5$ receptors induces late-phase LTP of C-fiber-evoked field potentials in rat spinal dorsal horn. J. Neurophysiol. 94, 961-967. doi: 10.1152/jn.01324. 2004

Yokogawa, F., Kiuchi, Y., Ishikawa, Y., Otsuka, N., Masuda, Y., Oguchi, K., et al. (2002). An investigation of monoamine receptors involved in antinociceptive effects of antidepressants. Anesth. Analg. 95, 163-168, table of contents.

Yoshida, A., Mobarakeh, J. I., Sakurai, E., Sakurada, S., Orito, T., Kuramasu, A., et al. (2005). Intrathecally-administered histamine facilitates nociception through tachykinin $\mathrm{NK} 1$ and histamine $\mathrm{H} 1$ receptors: a study in histidine decarboxylase gene knockout mice. Eur. J. Pharmacol. 522, 55-62. doi: 10.1016/ j.ejphar.2005.08.037

Yu, J., Tang, Y. Y., Wang, R. R., Lou, G. D., Hu, T. T., Hou, W. W., et al. (2016). A critical time window for the analgesic effect of central histamine in the partial sciatic ligation model of neuropathic pain. J. Neuroinflammation 13:163. doi: 10.1186/s12974-016-0637-0

Zhang, X. Y., Yu, L., Zhuang, Q. X., Peng, S. Y., Zhu, J. N., and Wang, J. J. (2013). Postsynaptic mechanisms underlying the excitatory action of histamine on medial vestibular nucleus neurons in rats. Br. J. Pharmacol. 170, 156-169. doi: $10.1111 /$ bph. 12256 
Zhang, Y. Q., Gao, X., Ji, G. C., Huang, Y. L., Wu, G. C., and Zhao, Z. Q. (2002). Expression of 5-HT1A receptor mRNA in rat lumbar spinal dorsal horn neurons after peripheral inflammation. Pain 98, 287-295. doi: 10.1016/s03043959(02)00026-X

Zhang, Y. Q., Gao, X., Ji, G. C., and Wu, G. C. (2001). Expression of 5-HT2A receptor mRNA in rat spinal dorsal horn and some nuclei of brainstem after peripheral inflammation. Brain Res. 900, 146-151. doi: 10.1016/s0006-8993(01) 02283- 1

Zorman, G., Hentall, I. D., Adams, J. E., and Fields, H. L. (1981). Naloxonereversible analgesia produced by microstimulation in the rat medulla. Brain Res. 219, 137-148. doi: 10.1016/0006-8993(81)90273-0
Conflict of Interest: The authors declare that the research was conducted in the absence of any commercial or financial relationships that could be construed as a potential conflict of interest.

Copyright (c) 2019 Bravo, Llorca-Torralba, Berrocoso and Micó. This is an openaccess article distributed under the terms of the Creative Commons Attribution License (CC BY). The use, distribution or reproduction in other forums is permitted, provided the original author(s) and the copyright owner(s) are credited and that the original publication in this journal is cited, in accordance with accepted academic practice. No use, distribution or reproduction is permitted which does not comply with these terms. 